
等道的今
PREPARED FOR THE U.S. DEPARTMENT OF ENERGY, UNDER CONTRACT DE-AC02-76-CHO-3073

PPPL-2868

PPPL-2868

UC- $420,421,426$

ION CYCLOTRON TRANSMISSION SPECTROSCOPY

IN THE TOKAMAK FUSION TEST REACTOR

BY

G.J. GREENE

SEPTEMBER, 1993
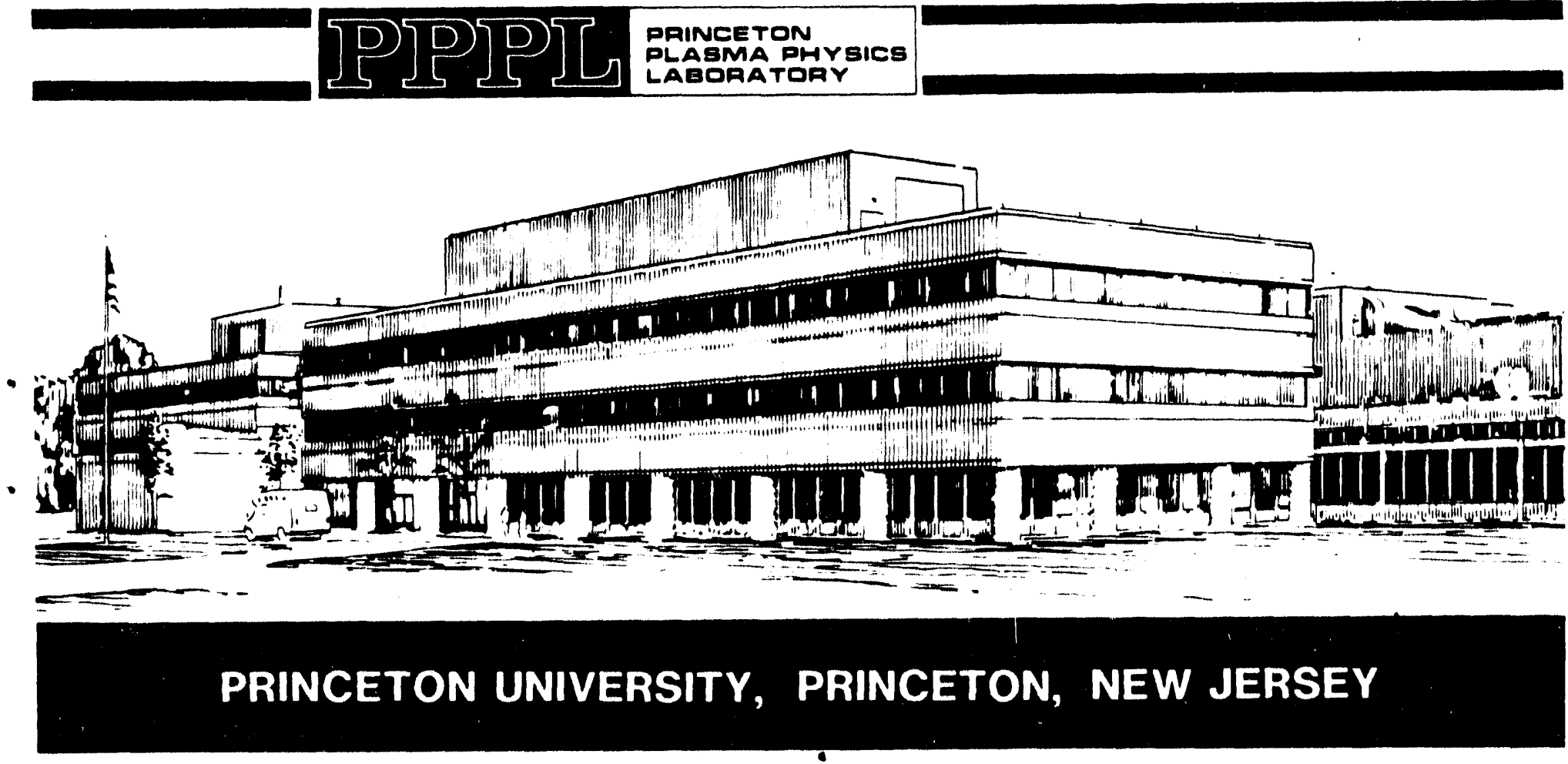

PRINCETON UNIVERSITY, PRINCETON, NEW JERSEY 


\section{NOTICE}

This report was prepared as an account of work sponsored by an agency of the United States Government. Neither the United States Government nor any agency thereof, nor any of their employees, makes any warranty, express or implied, or assumes any legal liability or responsibility for the accuracy, completeness, or usefulness of any information, apparatus, product, or process disclosed, or represents that its use would not infringe privately owned rights. Reference herein to any specific commercial produce, process, or service by trade name, trademark, manufacturer, or otherwise, does not necessarily constitute or imply its endorsement, recommendation, or favoring by the United States Government or any agency thereof. The views and opinions of authors expressed herein do not necessarily state or reflect those of the United States Government or any agency thereot.

\section{NOTICE}

This report has been reproduced from the best available copy.

Available in paper copy and microfiche.

Number of page in this report: 54

DOE and DOE contractors can obtain copies of this report from:

Office of Scientific and Technical Information

P.O. Box 62

Oak Ridge, TN 37831;

(615) $576-8401$.

This report is publicly available from the:

National Technical Information Service

Department of Commerce

5285 Port Royal Road

Springfield, Virginia 22161

(703) $487-4650$ 


\title{
Ion Cyclotron Transmission Spectroscopy in the Tokamak Fusion Test Reactor
}

\author{
G.J. Greene* \\ Princeton Plasma Physics Laboratory \\ P.O. Box 451, Princeton, New Jersey 08543
}

\begin{abstract}
The propagation of waves in the ion cyclotron range of frequencies has been investigated experimentally in the Tokamak Fusion Test Reactor. A small, broadband. radiofrequency ( $\mathrm{rf}$ ) magnetic probe located outside the plasma limiter, at a major radius near that of the plasma center, was excited with a low power, frequency swept source (1-200 . $\mathrm{MHz}$ ). Waves propagating to a distant location were detected with a second, identical probe. The $\mathrm{f}$ transmission spectrum revealed a region of at tenuation over a band of frequencies for which the minority fundamental resonance was located between the outer plasma edge and the major radius of the probe location. Distinct, non-overlapping attenuation bands were observed from hydrogen and helium-3 minority species; a distinct tritium band should be observed in future DT experiments. Rapid spectrum acquisition during a helium-3 gas puff experiment showed that the wave attenuation involved the plasma core and was not a surface effect. A model in which the received power varied exponentially with the minority density, averaged over the resonance region, fit the time evolution of the probe signal relatively well. Estimation of a 1-d tunneling parameter from the experimental observations is discussed. Minority concentrations of less than $0.5 \%$ can be resolved with this measurement.
\end{abstract}

* Present address: University of Pennsylvania School of Medicine, Box 419 Stemmler Hall, Philadelphia, Pennsylvania, 19104 


\section{$\therefore$ INTRODUCTION}

The propagation of waves in the ion cyclotron range of frequencies (ICRF) in a tokamak plasma is of considerable current interest. Most previous experimental work in this area has been motivated by applications of wave damping at high power levels for plasma heating. In this paper, we present measurements of low power wave propagation in a large tokamak with potential diagnostic utility as well as relevance for the evaluation of present theoretical models.

The earliest experimental studies of ICRF wave propagation in a tokamak were performed in the TM-1- V ch and TO-1 tokamaks $[1,2]$ using a coupling loop and a magnetic probe. A number of related efforts in small tokamaks followed $3-5$. In these experiments, an antenna, frequently consisting of a poloidal current element centered at the low-field midplane side of the plasma, launched waves thought to be primarily on the fast wave branch of cold plasma theory. The wave damping per toroidal and poloidal pass around the vessel was small, and consequently the notable experimental features were eigenmodes with large resonant enhancement of the wave fields at various values of plasma density, frequency, and toroidal field. It was recognized that the number density of these fast wave eigenmodes (per unit interval in plasma density, frequency, or toroidal field) increased rapidly with plasma density and with the vessel size. Indeed, in the current generation of large tokamaks (TFTR, JET, JT-60), a near continuum of modes would be expected at normal operating densities 6 ].

High frequency magnetic probes were used in some early studies to directly detect propagating waves in the plasma. The incident heat flux was sufficiently low that a probe could be inserted a significant fraction of the minor radius past the limiter of the tokamak without disturbing gross plasma parameters. Several experiments in small, low temperature tokamak plasmas mapped radial profiles of eigenmode field components $\{--9\}$ and found varying agreement with eigenmode theory.

A fundamental difference in wave propagation was observed with the general tendency in later and larger tokamaks to use deuterium as the primary gas species rather than hydrogen. Operation in relatively pure hydrogen $(H)$ plasmas had been possible, 
but nominal operation in deuterium $(D)$ was nearly always accompanied by a minor. ity hydrogen concentiation, and this impurity significantly altered the experimental results. It was first observed on the TFR tokamak 10 , 11 that in a $D$ majority, $H$ minority plasma. the presence of the proton fundamental resonance layer in the plasma led to significant damping of the wave fields and disappearance of eigenmodes. In these experiments, an ICRF heating antenna on the low field side of the tokamak was excited and signals on magnetic probes in the shadow of the limiter (apparently also on the low field side) were recorded. The toroidal magnetic field was varied on a shot by shot basis to scan the resonance layers across the plasma. With a fractional hydrogen concentration $n_{H} / n_{D} \simeq 0.2$, a significant asymmetry was found in the wave amplitude about the plasma center: attenuation was significant when the proton resonance layer was located at major radii between $R_{p}-(0.2) a_{p}$ and $R_{p}-(0.9) a_{p}$, where $R_{p}$ and $a_{p}$ are the plasma major and minor radii, respectively.

The asymmetric damping in TFR was explained by Jacquinot, et al., in terms of mode conversion from the fast magnetosonic wave to a slow, electrostatic wave near the two-ion hybrid layer $12 \mid$. The wave propagation equation near the hybrid layer was reduced to the form discussed by Budden [13], with the now familiar result that the fate of wave energy incident on the mode conversion zone is markedly different depending on the side of approach. This TFR study assumed that damping of the wave in a single pass through the resonance and mode conversion regions was small for incidence from either side. To our knowledge, this work, reported in 1977, remains unique in showing good agreement between the form of the fast wave fields as a function of resonance layer position and the predictions of a model.

A further discussion of related TFR experiments with varying isotopic ratios was given subsequently by Lapierre 14]. Here, results presented for $n_{H} / n_{D} \simeq 0.05$ and 0.25 were qualitatively different from those found in the earlier reference (\{12\}). For the case with lower minority concentration, the asymmetry was less pronounced and the wave field minirnum occured essentially when the resonance layer was at the plasma center. For the higher concentration case (closer to the parameters for the experiment 
discussed in reference 12 ), an apparent double minimum was found as the resonance layer was scanned across the plasma. These results were not explained by the mode conversion theory discussed in the reference.

In a later experiment on the diverted tokamak DIVA with a $D$ majority, $H$ minority plasma. the resonance layer was also scanned across the plasma by varying the toroidal field from shot to shot [15. Here a low field side half-turn loop antenna was used to launch waves, and a magnetic probe, apparently also located on the low field side but displaced toroidally, measured the radiofrequency ( $\mathrm{rf}$ ) wave field $B_{\theta}$. For an isotopic ratio $n_{H} / n_{D} \simeq 0.05-0.10$, asymmetric attenuation of the wave field was observed with respect to the position of the hybrid layer relative to the plasma center and was also attributed to mode conversion. The form of the attenuation data as a function of resonance layer position showed a weak minimum between the plasma center and the outside edge and, as in reference 14, the field amplitude (for resonance layer positions outboard of the plasma center) rose again for the single toroidal field data point at which the resonance laver was outside the plasma. The mode conversion model invoked included multiple reflections of waves between the cutoffs at the inside and outside plasma boundaries and absorption, transmission (tunneling), and mode conversion at the two ion hybrid laver. The predicted wave field variation with the position of the resonance layer, however, was significantly different from the experimental data.

A recent sequence of novel experiments on a small tokamak (TORTLS) revealed waves that propagated in the plasma edge region during ICRF excitation by a variety of antennas 16,17 . High-m poloidal modes, produced by a poloidally localized source, were shown to produce a spatially localized, guided beam of waves that propagated in the edge region along the direction of the total magnetic field. Other evidence for localized, guided propagation in the edge region was found on TEXTOR and ASDEX 18 and on the Caltech tokamak [19]. These experiments suggest that related phenomena may go undetected in large tokamak ICRF heating experiments for lack of suitable wave field diagnostics. 
Laser scattering experiments offer non-invasive, localized measurements but have been used in only a few instances to study ICRF wave propagation in the plasma interior. Evidence for mode-converted electrostatic waves was found near the two-ion hybrid resonance in the Microtor tokamak 20, and an experiment on TFR in addition observed the fast wave through its coupling to electrostatic drift waves 21. Further scattering experiments on Microtor 22 directly measured the density fluctuation associated with the fast wave itself (rather than the mode converted wave).

In recent years, there has been a large quantity of theoretical work directed toward understanding ICRF wave propagation 23,24 ]; there has been, conversely relatively little direct experimental data from modern, large tokamaks. Comparisons between theory and experiment have generally taken the indirect form of antenna loading calculations and heating models, in which the ICRF wave propagation is embedded in a much larger theoretical framework. The diagnostic difficulty of measuring internal rf wave fields remains a significant impediment to the verification of wave propagation theories. To our knowledge, scattering experiments to examine ICRF waves have not been successfully carried out in TFTR, JET, or JT-60. Similarly, radiofrequency probes have been infrequently used in these machines because of the inability to insert a probe into the bulk discharge and the significant risk of damage. Probes located far from the plasma have significantly weaker coupling and require more attention to the signal processing system and considerable care in interpretation of the data.

In this paper, we describe a simple experiment to examine low power ICRF wave propagation between two smail probes, located $0.1 \mathrm{~m}$ outside the limiter radius, in the Tokamak Fusion Test Reactor (TFTR). One probe was positioned roughly on the top of the plasma (at $R \simeq R_{p}$ ) while the second, displaced toroidally nearly a third of the way around the vessel, was located close to bottom of the plasma (also at $R \simeq R_{p}$ ). The geometry differs significantly from the usual ICRF heating scenario, and this choice, it will be seen, results in an unusual diagnostic possibility.

Rather than vary the toroidal field from shot to shot, as in experiments discussed above, the excitation frequency here was swept repetitively during a single discharge, 
effectively sweeping various resonance lavers across the entire plasma cross section. The rapid time response of the system allowed following the dynamics of the transmitted spectrum during perturbations with short gas puffs. A significant operational advantage was also realized through the independence of the experimental apparatus from the ICRF heating system.

The experimental results show the hallmarks of asymmetrical wave at teisuation associated with the effects of the two-ion hybrid layer and provide strong evidence that the attenuation occurs in the piasma core, not at the surface. The measurement of the transmitted spectrum was found to be useful as a diagnostic of minority ion concentration. It is hoped that the data presented here will also be of use in efforts to validate the 3-dimensional, global wave propagation models currently used in heating studies 25 '.

\section{EXPERIMENTAL APPROACH}

An array of wide bandwidth of magnetic probes was previously installed in TFTR at different toroidal and poloidal locations [26]. These probes were originally designed to detect spontaneus ion cyclotron emission from the plasma as well as waves launched by the high power (multi-megawatt) ICRF heating system. Due to the proximity of some of the probes to the heating antennas and the requirements for robust construction in preparation for the DT experimental phase on TFTR, the probes were designed to safely handle a received power level of $1 \mathrm{~kW}$ for $2 \mathrm{~s}$. Consequently, these probes were also capable of acting as transmitting antennas at low power levels.

Each probe consisted of a pair of orthogonal single-turn rectangular loops (area $=1.2 \times 10^{-3} \mathrm{~m}^{2}$ ) oriented to measure the of magnetic field components $B_{\theta}$ and $B_{\phi}$, where $\theta$ and $\phi$ correspond to the poloidal and toroidal coordinates, respectively. Careful electrical balancing of the loops and the use of ceramic or slot ted metal shields effectively reduced electrostatic signals 27 . The experiments reported here utilized a pair of probes that were separated toroidally by $126^{\circ}$, with the $B_{\phi}$ loop of one probe 
used for transmitting and the $B_{\phi}$ and $B_{\theta}$ loops of the other probe used for signal detection. One probe, located toroidally at Bay T (the TFTR vacuum vessel is divided into 20 toroidal segments, labeled alphabetically as Bays A - T), was positioned near the top of the plasma: $R=2.48 \mathrm{~m}, Z=1.05 \mathrm{~m}$, where $R$ and $Z$ are the major radius and the height above the torus midplane. The second probe, at Bay M, was near the bottom of the plasma: $R=2.54 \mathrm{~m}, \mathrm{Z}=-1.05 \mathrm{~m}$. The vacuum vessel major and minor radii were 2.65 and $1.14 \mathrm{~m}$, respectively, and the poloidal limiters nearest the probes had major and minor radii of 2.65 and $0.95 \mathrm{~m}$, respectively. Ordinarily, the Bay T probe was used for transmitting and the Bay $M$ probe, for receiving, but several experiments reversed the pair with essentially the same results.

A schematic of the overall experimental system is shown in Fig. 1. In order to maximize experimental flexibility, both the of source and the detection and digitization electronics were located in the TFTR control room, some $300 \mathrm{~m}$ from the tokamak. Either a spectrum analyzer tracking generator or the output of a network analyzer was used as the swept of source; the signal was transmitted over a wide-band (500) $\mathrm{MHz}$ ) analog fiber-optic link to the tokamak area. There, the signal was amplified to a level of $10 \mathrm{~W}$, split into two components of equal amplitude but shifted by 180 degrees in phase (using a wide-band hybrid combiner), and applied to one probe through a pair of balanced coaxial lines. Note that in order to retain a very wide bandwidth, no impedance matching was used between the amplifier and the probe. Consequently, the loading of the probe was negligible and the probe loop excitation current was independent of the plasma.

Signals transmitted through the plasma and received with the second probe were amplified in the tokamak area (after combining the balanced output signals from each end of the loop, again with a 180 degree phase shift) with a series of remotely programmable wide-band of amplifiers and attenuators. The signal was then returned to the control room via a second analog optical link, where it was detected with the spectrum or network analyzer.

The probes and their associated signal processing electronics had a nominal use- 
ful frequency response of $1 \cdots 500 . \mathrm{HHz}$. Calibration of the probe characteristic was performed using a $0.23 \mathrm{~m}$ o.d., 50 ohm transmission line section equipped with a port for mounting a probe. As a receiver. the response of the system was well modeled with a simple transmission line analysis, gielding

$$
B_{-}=\frac{\sqrt{2} V_{p} e^{\alpha s} Z_{0}+Z_{a} / 2}{\omega A Z_{0}},
$$

where $B_{-}$is the root-mean-square (rms) magnetic field perpendicular to the plane of the probe loop. $l_{p}$ is the rms output voltage measured at the summation port of the hybrid combiner, $A$ is the loop area, $\omega$ is the angular frequency, $\alpha$ is the at tenuation factor for the coaxial cables at tached to the probe loop ends (which is frequency dependent due to the skin effect), $s$ is the length of those cables, and $Z_{0}$ is the characteristic impedance terminating the cables. Here $Z_{a}=i w l_{a n t}+R_{a n t}$ is a lumped-element model of the complex impedance of the loop, where $L_{a n t}$ is the loop inductance and $R_{\text {ant }}$, the frequency-dependent loop resistance.

Similarly, it can be shown that the rms probe loop current $\left(I_{a}\right)$ is related to the rms forward voltage at the output of the generator $\left(l_{g}\right)$ by

$$
I_{a}=\frac{\sqrt{2} V_{g} e^{-a s}}{Z_{0}+Z_{a} / 2}
$$

Must if the experimental results reported in this paper are presented as a transfer func. tion $T$. defined here as the magnetic field (in gauss) at the second probe, normadized by the lowp current (in amperes) in the first probe:

$$
T=B_{-} / I_{a}=\frac{l_{p} e^{2 a} \cdot Z_{0}+Z_{a} /\left.2\right|^{2}}{l_{y}}
$$

In order to provide adequate time resolution during transient gas puff experiments. it was important to maximize the allowable sweep rate of the rf source. Ordinarily, the sweep rate of a spectrum or network analyzer is limited by the intermediate frequency bandwidth: however, an additional complication arose in this experiment. The length of the optical and of coaxial cables resulted in a round-trip signal propagation time, from source to detector, of about $3.5 \mu \mathrm{sec}$. At high sweep speeds, this time lag can 
result in the returned signal moving entirely outside the analyzer frequency window; this condition occurs when $\tau_{s}<2\left(f_{2}-f_{1}\right) \tau_{p} / H$. where $\tau_{s}$ is the sweep period, $\tau_{p}$ is the round-trip propagation delay, $\mathbb{I}$ is the analyzer bandwidth, and $f_{2}$ and $f_{2}$ are the starting and ending frequencies for the sweep. Therefore, increasing the detection bandwidth was desirable, although at the expense of an increased noise floor. For parameters typical of these experiments, the system could record a spectrum every $5.6 \mathrm{msec}$ during a tokamak discharge with negligible signal distortion; this provided adequate time resolution for the transient gas puff experiments discussed below. In general, data was taken with fast time resolution using the spectrum analyzer and tracking generator combination as a signal source and detector due to the faster sweep rates and wider signal bandwidth available; the network analyzer, with its narrower signal bandwidth, was used for long sweep periods when the discharge was stable.

\section{RESULTS AND DISCUSSION}

In this section, we present and discuss data from a series of recent experiments in TFTR. The data were taken in the ohmic phase of tokamak discharges, prior to any neutral beam or ICRF heating. Deuterium and helium-3 discharges were used. in sone cases with a short gas puff of helium-3.

The spectra of cavity modes that are observed in the absence of any plasma are described in Sec. 3.1. Experiments with a deuterium majority, hydrogen minority plasma are presented in Sec. 3.2. The rather different spectra obtained in discharges that began with a prefill of helium-3 are described in Sec. 3.3, as are data obtained pertaining to the wave polarization. Perturbation of a deuterium majority, hydrogen minority plasma with a small gas puff of helium-3 and comparisons of the spectrum and density evolution are topics of Sec. 3.4. Finally, the relation of the experimental observations to the tunneling parameter of a simple one-dimensional model is discussed in Sec. 3.5. 


\subsection{Transmission in vacumm}

A transmission spectrum with distinct and const ant features was always observed before anci after a plasma discharge in TFTR. In the absence of plasma. the rac unm vessel effectively forms a toroidal cavity resonator. This cavity is cut off (the wavenumber becomes imaginary) below a particular frequency that depends on the toroidal geometry; above that frequency a variety of modes can propagate. Excitation at high power of such vacuum cavity modes for the purpose of heating the surface of tiles lining the racuum ressel of tokamak reactors (via joule heating from the induced surface currents) has been proposed by Colestock 28. Although the theoretical analy sis of even this simple, plasma-free system is very complex 29|, these modes were clearly observed here, apparently for the first time. The spectrum of Fig. 2 plots the received probe power as a function of frequency and shows that the cavity is cut off below about $78 . \mathrm{MHz}$. Above the cutoff frequency, a series of modes are apparent which form almost a continuum at higher frequencies. We note that the observed cutoff fre quency in lower by about $20 \%$ than the value calculated from the results of reference 30) and the spacing between the first few modes is also considerably smaller than predicted. The system noise foor is at about $72 \mathrm{dbm}$, and the small peaks observed below 50 $.11 \mathrm{~Hz}$ are probably due to rf leakage from the vessel or cables.

\subsection{Deuterium majority, hydrogen minority plasmas}

During an ohmic plasma discharge, the transmission spetrum changes dramatically from the vacuum case. The spectrum shown in Fig. 3 was taken during the steady state (current and field flat-top phase) of a deuterium discharge. For this discharge, the major radius $\left(R_{p}\right)$ of the plasma was $2.58 \mathrm{~m}$, the minor radius $\left(a_{p}\right)$ was $0.93 \mathrm{~m}$, the

plasma current $\left(I_{p}\right)$ was $1.19 \mathrm{MA}$, the toroidal field $\left(B_{T F}\right)$ at a major radius of 2.65 $\mathrm{m}$ was $3.69 \mathrm{~T}$, the central vertical chord integrated electron density $\left(\overline{n_{e} \ell}\right)$ was $3.9 \times$ $10^{19} \mathrm{~m}^{2}$, and the central electron temperature $\left(T_{e}\right)$ was $2.0 \mathrm{keV}$. (In this paper, the toroidal field is specified at the vessel center, while values of $n_{e} k$ and $T_{e}$ refer to the plasma center unless otherwise noted.) In similar ohmic discharges, the proton minor- 
ity concentration, estimated on the basis of ICRF heating data, is typically thought to be in the range of $4-8 \%$ (although spectroscopic estimates are generally higher), and ion and electron temperatures are similar to each other. The data, displayed as the transfer function $T$ defined above, represents the average of $V_{s}=9$ consecutive frequency sweeps within a single plasma discharge. each with a duration $\left(\tau_{s}\right)$ of 130 ms. Including the retrace time between sweeps, the displayed data comes from a time window (-w) of $1.6 \mathrm{~s}$.

A prominent drop is seen in the signal at frequencies between 40 and $60 \mathrm{MHz}$, and a second apparent dip occurs between 10 and $25 \mathrm{MHz}$. Three vertical dashed lines are plotted at the frequencies for which the proton minority cyclotron resonance layer is positioned at the major radius of the outer plasma edge (i.e., at $R=R_{p}+a_{p}=3.51 \mathrm{~m}$ ), at the transmitting probe $(R=2.48 \mathrm{~m})$, and at the inner plasma edge $\left(R=R_{p}-a_{p}=\right.$ $1.65 \mathrm{~m})$. The large decrease in signal transmission occuring in the frequency band for which the proton resonance layer is located at major radii between the outer plasma edge and the transmitting probe contrasts with the much larger transmission in the interval fur which the resonance layer is located between the major radii of the probe and that of the inner plasma edge. The difference in transmission in the two bands corresponds to an attenuation factor of about 25 in wave amplitude.

The smaller dip in the transfer function $(T)$ between 10 and $25 \mathrm{MHz}$ in Fig. 3 is frequentiy not observed, while the rise at low frequencies is always present. In fact, the inr rease in signal below $10 . \mathrm{MHz}$ is due to a particular kind of experimental noise. The plasma itself emits radiation in the ICRF band and a low frequency continuum emission was previously observed from TFTR 31\%. An experiment was performed to assess the relative magnitudes of the signals from spontaneous plasma emission and the driven RF probe. The received signal was split into two equal components with a hybrid junction, and a second spectrum analyzer, synchronized in time but offset in frequency (by $-5 \mathrm{MHz}$ ) was used to record the spontaneous emission simultaneously with the usual transmission signal. Separate tests confirmed that broadening of the driven waves in frequency was negligible compared to the frequency offset of the second 
analyzer.

A simultaneous measurement of the emission and transmission for a deuterium discharge is shown in Fig. 4. The relevant parameters for the discharge were $R_{p}=2.61$ m. $a_{p}=0.95 \mathrm{~m}, I_{p}=1.58 . \mathrm{AA}, B_{T F}=4.44 \mathrm{~T}, n_{e} \ell=4.3 \times 10^{19} \mathrm{~m}^{-2}, T_{e}=2.5 \mathrm{kel}$, and the signal acquisition parameters were $\tau_{s}=5 \mathrm{~ms}, V_{s}=9$, and $\tau_{w}=50 \mathrm{~ms}$. At frequencies below about $12 . \mathrm{HHz}$, the spontaneous plasma emission alone produces a signal that is essentially unchanged by the addition of active probe excitation. Hence, we conclude that spontaneous emission, rather than the driven RF wave, accounts for the low frequency rise in the data. The contribution to the spectrum arising from active wave excitation cannot be determined in this low frequency region. Also plotted in the figure is the background noise level of the system; this represents largely the noise level associated with the broadband of analog optical links. This noise level is simificantly below the transmission signal level over the entire frequency range. As before, the data shown has been normalized and calibrated and is presented as the transfer function $T$.

Dinte that the transmission spectrum of Fig. 4 shows considerably more fluctuation than the data in Fig. 3. The data for the spectrum in Fig. 4 were taken at a much faster sweep speed (5 ms vs. $130 \mathrm{~ms}$ ) and over a much shorter total time interval (50 ms vs. $1.6 \mathrm{~s})$. The fluctuations are believed to represent modes, i.e., the constructive and destructive interference of waves reflecting from various features in the plasma or boundary as the frequency or plasma parameters (such as density) change. These modes maintain similar shape over several or many successive frequency sweeps, suggesting that they do not arise from plasma fluctuations (since it is unlikely such a fluctuation would be locked to the spectrum analyzer sweep period). The modes usually become more prominent as the time resolution of the transmission measurement is increased by using faster and fewer frequency sweeps. These modes may be averaged or 'washed out' in data taken over longer time intervals (e.g., the spectrum in Fig. 3) if, for instance, the plasma density drifts slightly during the averaging window so that the frequency at which each mode appears is shifted by a 
significant amount compared to the width of the mode.

The cyclotron origin of the attenuation phenomena observed in Fig. 3 was verified by examining shots with different toroidal magnetic field values. Transmission spectra are shown in Figs. $5(a)$ and $5(b)$ for the extrema in toroidal field for which spectra were obtained: the vertical dashed lines again show the frequencies at which the proton resonance laver is at the outer plasma edge, the probe location, and the inner plasma edge. The plasma conditions for these two deuterium discharges were different. For the high field case of Fig. $5(\mathrm{a}), B_{T F}=4.45 \mathrm{~T}, I_{p}=2.00 \mathrm{MA}, \overline{n_{e} \ell}=4.3 \times 10^{19} \mathrm{~m}^{-2}$, and $T_{e}=3.4 \mathrm{keV}$. For the low field discharge (Fig. $5(\mathrm{~b})$ ), $B_{T F}=2.00 \mathrm{~T}, I_{p}=0.89$ $11 \mathrm{~A}, \overline{n_{e} \ell}=3.5 \times 10^{19} \mathrm{~m}^{-2}$, and $T_{e}=1.4 \mathrm{keV}$. For both discharges, $R_{p}=2.45 \mathrm{~m}$, $a_{p}=0.80 \mathrm{~m}$, and the signal acquisition parameters were $\tau_{s}=240 \mathrm{~ms}, N_{s}=3$, and $\tau_{w}=0.83 \mathrm{~s}$. In both of these cases (as well as the intermediate field case shown in Fig. 3), a large drop in signal transmission occurs when the proton resonance layer is located approximately between the outer plasma edge and the probe location. Note also that in both Fig. 3 (at $\sim 92 \mathrm{MHz}$ ) and in Fig. $5(\mathrm{~b})($ at $\sim 62 \mathrm{MHz}$ ), there is a very small decrease in transmission. This phenomena is occasionally but not always seen; the observed frequencies suggest that it could involve second harmonic damping by protons.

The cold plasma wave dispersion surfaces $[32 \mid$ are plotted in Figs. 6(a) and 6(b) for the plasma conditions of the discharge discussed above in association with Fig. 3. Surfaces are shown for two different frequencies ( 50 and $65 \mathrm{MHz}$ ), corresponding to the region of maximum attenuation and an adjacent region of significant transmission. The fast magnetosonic wave cutoff occuring at $n_{1}^{2}=S+D$ (where $S$ and $D$ are the elements of the cold plasma dielectric tensor as defined by Stix $\left[33 i\right.$, and $n_{\|}$is the index of refraction parallel to the toroidal magnetic field) is nearly circular; the minority cyclotron resonance layer is of course vertical. The two-ion hybrid resonance $\left(n^{2}=S\right)$ and associated cutoff $\left(n_{\|}^{2}=S-D\right)$ lie close together and are nearly vertical within the interior (propagating region) of the $n_{\|}^{2}=S+D$ cutoff. The width of the mode conversion region between the hybrid resonance and cutoff pair depends 
on the minority concentration but is typically less than a few centimeters for TFTR conditions; similarly, the displacement of the mode conversion region to the high field side of the minority cyclotron laver is typically less than 10 centimeters. The surfaces shown are only illustrative: they are plotted for a hydrogen ion concentration of $n_{H} / n_{D}$ $=0.05$ and for a value of the parallel wavenumber $(k)$ fixed at $7 \mathrm{~m}^{-1}$. It is likely, of course, that radiation from the small probe includes a broad spectrum of wavenumbers. Note that the small frequency shift $(<2 \mathrm{MHz})$ associated with the difference in radial position between the minority cyclotron and mode conversion regions cannot be readily observed in the experimental data. Hence, in this paper, comparisons are made with the locations of the cyclotron resonance layers.

\subsection{Multiple minority species}

The observation that the regions of pronounced signal attenuation in the deuterium majority, hydrogen minority case are confined to those frequencies for which the minority cyclotron resonance laver lies between the probe location and the outer plasma boundary suggests that more than one minority species might be detectable. If the attenuation depends only on the presence of a minority layer between the probe and the outer plasma edge, than it follows that attenuation bands from two different minority species will be completely resolved (i.e., will not overlap) provided

$$
\Gamma \equiv \frac{m_{2} q_{1}}{m_{1} q_{2}}>\frac{R_{p}+a_{p}}{R_{p r}} \approx 1+\epsilon \approx 1.3
$$

where $m_{i}$ and $q_{i}$ are the mass and charge of minority species $i(i=1,2)$, species 1 has the greater charge to mass ratio, $R_{p r}$ is the major radius of the probe location (which is near $R_{p}$ ), and $\epsilon$ is the inverse aspect ratio of the plasma. Evaluation of the above shows that for hydrogen and helium-3 minority species, $\Gamma=1.5$ : for helium-3 and tritium, $\Gamma=2$. Hence it follows that absorption bands from hydrogen, helium -3 and tritium minority species should all be distinct. Note that this resolution crucially depends on the localization of attenuation to the outer region $\left(R>R_{p r}\right)$ of the plasma: if attenuation occured whenever the minority resonance layer was inside the plasma 
boundary (i.e., between $R_{p}+a_{p}$ and $R_{p}-a_{p}$ ), then the attenuation bands for the above species would in fact overlap.

While tritium has not yet been used in TFTR, helium-3 is frequently introduced by gas puffing in conjunction with ICRF heating experiments. During such periods, recycling of deuterium from the vessel walls and limiters is reduced (after a disruption, for instance) using a sequence of 'conditioning' shots in which a small amount of helium-3 is introduced before initiation of the plasma and no external gas puffing is employed. In such discharges. the helium-3 is used primarily for the initial breakdown phase, following which the plasma is fueled from gas evolving from the walls and limiters. The actual ratios of the component species ( $H, D, H e-3)$, however, are not readily measured.

The rf transmission spectrum from a typical helium-3 conditioning discharge is shown in Fig. 7 . The associated plasma parameters were: $B_{T F}=3.69 \mathrm{~T}, I_{p}=1.39$ MA, $R_{p}=2.45 \mathrm{~m}, a_{p}=0.80 \mathrm{~m}, \overline{n_{e} \ell}=4.8 \times 10^{19} \mathrm{~m}^{-2}$, and $T_{e}=2.1 \mathrm{keV}$. The spectra were taken with the network analyzer system at a slow sweep speed $\left(\tau_{s}=130\right.$ ms, $\left.V_{s}=\tau, \tau_{u}=1.24 \mathrm{~s}\right)$ during the 2 -s flattop of the discharge. Vertical dashed lines are plotted at the frequencies for which the hydrogen and helium-3 cyclotron resonances are located at the outer plasma edge $\left(R=R_{p}+a_{p}\right)$ and at the probe location $\left(R-R_{p r}\right)$. Two distinct dips in the transmission are evident and are largely localized to the regions over which the respective minority cyclotron resonances for hydrogen and helium-3 lie between the outer plasma edge and the probe location. Thus it appears that attenuation from the two minority species can in fact be observed separately. The precise shape of the transmission curve in the regions of attenuation, however, is somewhat variable. In the example shown here, the attenuation dip in the band associated with helium-3 is somewhat narrower than that in the hydrogen band; in other cases, this distinction is not seen.

For comparison, the cold plasma surfaces are plotted in Fig. 8 for the parameters of this conditioning discharge, assuming $n_{H} / n_{D}=n_{H e-3} / n_{D}=0.05$ and $k_{\|}=0.7$ $\mathrm{m}^{-1}$. A measure of the fractional helium-3 concentration in this type of discharge is 
not available; the assumed value here is roughly twice the value estimated for the gas puff discharges discussed below (note that $n_{\mathrm{He}-3}$ refers to the ion density). The frequency chosen for this example $(50 \mathrm{MHz})$ lies within the band identified with hydrogen minority attenuation: however, the helium-3 cyclotron and mode conversion regions are simultaneously within the plasma. We will show below that cyclotron and mode Conversion layers inboard of the probe location $\left(R<R_{p r}\right)$ do not affect attenuation from other such regions at major radii greater than that of the probe.

The received of probe signals were typically taken from the $B_{\phi}$ loop of each sensor. However, signals from the orthogonal $B_{\theta}$ loop were also available and were used to investigate the wave polarization. Examples of the spectra from both sensors of a single probe in a helium-3 conditioning discharge are shown in Figs. $9(\mathrm{a})$ and $9(\mathrm{~b})$. Plasma parameters for the shot were essentially unchanged from the shot associated with Fig. 7 , except that the total density was higher: $\overline{n_{e} \ell}=6.0 \times 10^{19} \mathrm{~m}^{-2}$, suggesting that the helium-3 fractional density could be lower for this shot. Coincidentally, the depths of the attenuation bands were also smaller for this case. The network analyzer system was used to obtain the data, with $\tau_{s}=130 \mathrm{msec}, N_{s}=3$, and $\tau_{w}=0.66 \mathrm{~s}$. The $B_{\theta}$ and $B_{\phi}$ spectra have similar shape, although the $B_{\theta}$ signal is somewhat smaller in amplitude. Analysis of the data shows that the ratio $B_{\theta} / B_{\phi}$ fluctuates between about 0.2 and 1 over the frequency range plotted. The signal minima in the hydrogen and helium-3 attenuation bands are deeper for the $B_{\theta}$ signal, and the corresponding high frequency edges of the band, where the resonance layers are near the major radius of the probe, extend to higher frequency than for the $B_{\theta}$ signal. The phase between the two components has not yet been successfully measured; there is significant phase noise present that may arise from plasma fluctuations or wave modes. Therefore, no conclusion can be drawn about the ellipticity of the transverse polarization of the wave fields. However, a survey of a number of shots yields the same results shown in the figure - both transverse components of the wave field are present at the receiving probe, the spectra have similar shape, and the magnitude of the $B_{\theta}$ component is generally somewhat smaller. 


\subsection{Helium-3 perturbation experiments}

The transmission spectra presented thus far vere all taken during the steady. state portion of discharges: the gross plasma parameters were essentially constant. In this section. we present data from perturbation experiments in which a small, shwrt gas puff of helium-3 was introduced into a deuterium majority discharge. A significant advantage of such experiments is that the electron density rise associated with the gas putf directly provides an estimate of the fractional helium-3 concentration. Several hundred milliseconds after the gas puff, high power ICRF heating was applied to some of the discharges. These data describe the evolution of the spectrum only until the start of the ICRF heating pulse. Because the ICRF generator frequency $(47 \mathrm{MHz})$ was well within the signal bandwidth of the probe system, fast coaxial relays were used to gate off the signal processing electronics to prevent damage from the high power signals received during the heating pulse.

The vertical chord integrated electron density (at $R=2.68 \mathrm{~m}$ ) for a discharge with a helium-3 gas puff and without ICRF heating is shown in Fig. 10. Associated plasma parameters for this shot were: $B_{T F}=4.45 \mathrm{~T}, I_{p}=1.39 \mathrm{MA}, R_{p}=2.60 \mathrm{~m}$, $a_{p}=19.95 \mathrm{~m}$, and $T_{e} \approx 2.4 \mathrm{keV}$. Here the gas flow rate into the vessel during the puff was $2.3 \mathrm{TL} / \mathrm{s}$, and the duration of the puff was $155 \mathrm{~ms}$. The associated density rise is clearly visible, beginning at $\sim 2.8 \mathrm{~s}$. If the observed rise is assumed to be due to electrons from helium-3, then the fractional chord-averaged helium-3 ion concent ration after the puff is on the order of $2.5 \%$ (the corresponding rise in electron density is about $5 \%)$. The transmission spectra obtained with the network analyzer system $(\tau$, $=130 \mathrm{~ms}, N_{\mathbf{A}}=3, \tau_{w}=500 \mathrm{~ms}$ ) within the two time intervals indicated by the dashed lines in Fig. 10 are shown in Figs. 11 (a) and 11 (b). The forms of the spectra obtained before and after the helium-3 gas puff are similar to those for deuterium majority, hydrogen minority discharges (Fig. 3) and for helium-3 conditioning discharges (Fig. 7), respectively. It is clear that the attenuation band at lower frequency appears only after the helium-3 gas puff. 


\subsubsection{Comparisons of spectrum and density evolution}

As discussed in Sec. 1, there is some reason to suspect that a very small RF antenna, such as the probe used here, might excite various surface waves. There is a significant radial current at the probe, and the small physical size makes excitation of high poloidal mode number waves likely; both of these effects are thought to lead to waves peaked near the surface. A transient gas puff experiment presented an opportunity to test this hypothesis. The characteristic time for an external helium-3 gas puff perturbation to diffuse into the plasma core region is on the order of $50-100 \mathrm{~ms}$ 34. The plasma surface, however, is perturbed on a faster time scale. An experiment was designed to follow the evolution of the wave transmission spectrum with sufficient time resolution to compare it with chord integrated electron density data from the vertically viewing, multi-channel, infrared interferometer diagnostic 'MIRI' 35]. Using a tracking generator as the signal source for wave excitation, and a fast $(500 \mathrm{kHz})$ signal digitization rate, a spectrum acquisition time of $5.6 \mathrm{msec}$ was possible and was adequate for comparison with the interferometer data.

In a first experiment, the size of a helium-3 gas puff was changed on two successive shots by increasing the flow rate from $0.75 \mathrm{TL} / \mathrm{s}$ to $1.5 \mathrm{TL} / \mathrm{s}$; the gas puff duration was $48 \mathrm{~ms}$ in both cases. The transmission spectrum taken some $200 \mathrm{msec}$ after the larger puff is shown in Fig. $12\left(T_{1}=5.6 \mathrm{~ms}, N_{s}=9, T_{w}=49 \mathrm{~ms}\right)$. Plasma parameters for these shots were: $B_{T F}=4.44 \mathrm{~T}, I_{p}=1.58 \mathrm{MA}, R_{p}=2.61 \mathrm{~m}, a_{p}=$ $0.96 \mathrm{~m}, n_{e} \ell=4.5 \times 10^{19} \mathrm{~m}^{2}$, and $T_{e}=2.6 \mathrm{keV}$. The general features of hydrogen and helium-3 attenuation bands that were discussed above can al o be seen in this spectrum, although the apparent modes are large. In an attempt to reduce the effect of these modes on the data analysis, each successive spectra was averaged over selected frequency windows as indicated in the figure. The relative center of the window in the hydrogen attenuation band was chosen to be near the minimum of the signal, and the width of the window was chosen to cover at least several modes. Transforming from frequency to hydrogen cyclotron layer position, the selected window spans major radii from $R_{p}-(0.25) a_{p}$ to $R_{p}+(0.55) a_{p}$. The same relative window was used in 
the helium-3 attenuation band with respect to the helium-3 cyclotron layer position. Note that the averaging was performed on the signal as plotted (i.e., on $\log (T)$ ): this choice is discussed in more detail below.

The resulting data using the helium-3 averaging window are displayed as func. tions of time in Fig. 13 for the two successive shots. Since there is one data point per spectrum in each averaging window, the time resolution of these plots is $5.6 \mathrm{msec}$. The signals are relatively constant during the $100 \mathrm{msec}$ preceding the gas puff. The perturbation due to the gas puff is clear in both cases, and, as a result of the averaging procedure, the signals are much smoother than might be expected from examination of the raw spectra. The characteristic time for the increase in attenuation after the gas puff is on the order of $60 \mathrm{msec}$. The signal derived from the hydrogen attenuation band during the larger of the two gas puffs is also displayed; it exhibits little change associated with the gas puff. This result demonstrates the independence of the two attenuation bands: the presence of a minority cyclotron resonance and mode conver. sion layer on the high field side of the probe does not affect at tenuation observed from such lavers on the low field side that are associated with another minority species.

Data from a different lischarge with a helium-3 gas puff is shown in Fig. 14. In this case, the plasma parameters were $B_{T F}=3.24 \mathrm{~T}, I_{p}=1.39 \mathrm{MA}, R_{p}=2.69 \mathrm{~m}, a_{p}=$ $0.90 \mathrm{~m}, n_{e} \ell=2.6 \times 10^{19} \mathrm{~m}^{-2}$, and $T_{e}=3.2 \mathrm{keV}$. Here the frequency averaging window also corresponded to major radii from $R_{p}+(0.25) a_{p}$ to $R_{p}+(0.55) a_{p}$. Following the gas puff, the signal decreases somewhat more slowly than for the preceding case: the characteristic time here is on the order of 100 msec. The signal from the outermost interferometer chord has also been plotted in the figure. This chord, located at $R$ $3.47 \mathrm{~m}$ is some $0.12 \mathrm{~m}$ on the high field side of the nominal plasma outer midplane edge. The density signal has been inverted and normalized to the range of the of probe signal for comparison of the relative waveforms. It is clear that the density at the plasma edge is perturbed much more rapidly after the gas puff than the probe signal - the characteristic time for the density to peak is on the order of $35 \mathrm{msec}$. Also, the waveforms are quite different. The density at the edge peaks rapidly as the incident 
neutral helium-3 is ionized and then drops as the gas is transported inward. This data strongly suggests that the rf waves observed here are not propagating only in the plasma edge: otherwise. we would expect the probe signal to be perturbed as fast as the edge density. The time scale of the perturbation in the probe signal is appropriate to penetration of the gas to the plasma core.

Some simple quantitative estimates were obtained from the relative magnitudes of the frequency averaged spectrum evolution for the large and small gas puff discharges described above (Fig. 13). It appeared likely that the observed wave attenuation arose from interactions at the cyclutron resunance or wo-ion hybrid lavers, which form nearly cylindrical surfaces and lie close together through most of the plasma. Therefore, the vertical chord integrated electron density data from an interferometer channel located at $R=2.99 \mathrm{~m}$, near the major radius that corresponded to the center of the frequency averaging window, were compared for the two shots. For example, at $t=2.7 \mathrm{~s}$, the chord integrated electron density increments associated with the two gus puffs (not shown in the figure) were $7.70 \times 10^{17}$ and $1.97 \times 10^{18} \mathrm{~m}^{-2}$; the total chord integrated density before the gas puff was $3.60 \times 10^{19} \mathrm{~m}^{-2}$. These changes corresponded to $2.1 \%$ and $5.5 \%$ of the pre puff values, respectively. At the same time, the values of $\log (T)$ for the two discharges decreased from prepuff levels of -6.41 to values of 6.62 and 6.97, respectively: The ratio of the electron density increment from the large gas puff to that from the small one was -2.6 ; the corresponding ratio of the change in $\log (T)$ yielded a similar value of -2.7 . In contrast, the associated ratio of the changes in $T$ itself (rather than $\log (T)$ ) was calculated to be 1.9 . Hence, although this mumerical example is only for a single time point, the result suggests a linear relation between the chord integrated minority species density and the logarithm of the transfer function $T$.

In order to reduce signal noise and scatter and the effect of modes, it was desirable to average the transmission spectrum over a wide frequency window. However, in a detailed comparison of the time evolution of the probe signal with the electron density, the averaging process must be considered further. We have speculated that the signal 
attenuation arises from wave effects at the minority and hybrid resonances lavers. which lie close together on a nearly cylindrical surface. It follows that the shape of the attenuation curve should contain radial information, and a comparison of the evolution of a frequency averaged spectral windew should properly be made with some average of the density over the corresponding radial window.

Prompted by the results discussed above, we write the signal in the helium-3 attemuation band coorresponding to the case of high field incidence on the resonance liveren as

$$
r(f)=\text { Ae } P N R \text {. }
$$

where

$$
V(R)=2 \pi R \int_{a_{p}}^{+a_{p}} \Delta n_{e}\left(R_{,} z\right) d z=2 \pi R \Delta n_{e} \ell(R)
$$

is the electron density arising from the minority species, integrated over the cylindrical resonance surface corresponding to the frequency $f, \Delta n_{\ell} \ell(R)$ is the chord integrated density increment (from the mimority species) as measured by the interferometer at radius $R$. and $A$ and $D$ are constarts. Averaging the probe signal over the frequency window $f_{1}$ th $f_{2}$ then yields

$$
\log T \quad \frac{1}{\left(f_{2} \cdots f_{1}\right)} \int_{f_{1}}^{f_{2}} \log T(f) d f \quad C-D \xi
$$

where

$$
\xi=\frac{2 \pi R_{0} f_{0}}{\left(f_{2}-f_{1}\right)} \int_{H_{1}}^{R_{1}} \frac{\Delta n_{e} \ell(R)}{R} d R .
$$

Here $G$ is a constant independent of $R$ and the minority density, and $f / f_{0}=R_{0} R$, where $R$ is the major radius associated with a helium 3 cyclotron resonance at fre. quency $f$. The subscript ' 0 ' refers to the vessel center, while the subscripts ' 1 ' and '2' represent the limits of the averaging interval. With this definition, $f_{0}-\mu B r F(2 \pi)$, where $\mu$ is the charge to mass ratio for helium-3. The left hand side of equation ( 7 ) is the experimental quantity derived from the transmission spectra as shown, for ex. ample, in Fig. 13. The right hand side of the equation was evaluated by interpolating data from the ten inteferometer channels to estimate the function $\Delta n_{e} \ell(R)$. In cases 
where the background density was slowly rising before the gas puff, the rate of rise was extrapolated and the resulting estimate of the background density was subtracted to vield the density from the gas puff alone.

An analysis of the discharge with the larger helium 3 gas puff fdiscussed in as. sociation with Fig. 13) was performed with an averaging window corresponding to a radial range for the helium-3 cyclot ron position of $R_{p}-(0.12) a_{p}$ to $R_{p}+(0.62) a_{p}$. This broad averaging window was chosen to cover a number of modes. A plot of the two sides of equation 7 is shown in Fig. 15. Here the constant ( $i$ was selected to match the pre-putf values of the functions. and a value of $D=1.67 \times 10^{-20} \mathrm{~m}$ then gave the best fit for the rest of the data. It is clear that the time evolution of the frequency averaged probe signal follows the density intent al rather closely. This result supports the above hypothesis that the signal attenuation varies exponentially with the minority density. averaged over the resonant surface.

\subsubsection{Compamson of two madial windous}

In the previous section, comparisons were made by integrating the frequency spec. tra over a range corresponding to half the major radius of the plasma. Here, we consider the signals obtained by averaging the spectra over narrower frequency windows.

If the previous empirical description of the wave attenuation is correct, then ra. dial profile information should be contained in the transmission spectra. It is clear, however, that Eq. (7) cannot hold over the entire half space between the plasma edge and the probe position, because the transmitted signal generally exhibits a minimum at frequencies corresponding to cyclotron resonance layer positions that are somewhat on the low field side of the major radius of the probe position, rather than at the probe position itself (where the value of the chord integrated density is usually near its maximum). Vevertheless, some conclusions can be drawn from a comparison of the time evolution of signals obtained by averaging different frequency bands.

The use of a smaller frequency averaging interval to examine the evolution of the spectrum increases the signal noise. In addition, if only one or a few modes are present 
within the selected window, the shifts of their individual frequencies as the density changes could obscure the interpretation of a gas puff experiment. Despite these caveats. a comparison of the density integral and the transmission signal ( $\mathrm{Eq} .7$ ) during a discharge with a helium.3 gas puff was made using two narrower windows located near the lower and upper frequency range of the attenuation band (corresponding to radial positions near the outside and center of the plasma, respectively). In terms of the helium-3 cyclotron laver position, the first frequency window corresponded to a range from $R_{p}$ to $R_{p}+(0.2) a_{p}$ (referred to below as the central window) and the second, from $R_{p}+(0.5) a_{p}$ to $R_{p}+(0.7) a_{p}$ (referred to as the outer window). The results are shown in Figs. 16(a) and 16(b), where the data ranges have been normalized to permit comparison of the relative time evolution of the waveforms. The transmission signal obtained using the central averaging window clearly lags the signal from the outer window, as do the corresponding density integrals. However, we note that the density signal obtained with the outer window drops more rapidly than the corresponding transmission signal, and the transmission signal using the cental window, while having a similar shape, slightly lags the corresponding density signal.

In the simplest view, the density integral corresponding to the central averaging window lags the signal obtained using the outer window because of the time required for the gas, applied externally, to penetrate to the plasma core. Integration along a vertica! chord near the plasma center also includes the edge regions at the top and bottom of the path, and the faster perturbation of the edge region contributes to the lack of an obvious time lag between the central and outer density integral signals (Fig. 16(b)). In contrast, the transmission data (Fig. 16(a)) does show an apparent time lag before the signal ubtained with the central averaging window begins to drop. This observation suggests that the process causing the wave attenuation may be more heavily weighted by the plasma core region than the simple chord integral of the density in Eq. (7) would suggest. Such an effect could arise, for instance, if the plasma temperature was a significant component in the wave propagation physics, since the temperature profile is peaked in the core. While investigations of the full two-dimensional evolution of 
the transmission spectra $(T(f, t))$ and detailed comparisons of the spectral shapes to radial profile data have just begun, the clear time delay in the two transmission signals obtained using averaging windows corresponding to different radial zones supports the idea that radial profile information is contained in the spectra the plasma center should be perturbed on a slower time scale than the outer regions.

\subsection{RELATION TO TUNNELING PARAMETER}

The actual wave propagation in this experiment is expected to be complex: the overall direction of propagation includes toroidal and vertical components that are tangential to the cyclotron and two-ion hybril surfaces. In addition, the probe is physically small and will launch a broad spectrum of wave vectors, and wavelengths can be significant compared to the characteristic dimensions of the tokamak. Nevertheless, some useful analogies may come from elements of a simpler model that considers the effect of the two-ion hubrid resonance-cutoff pair. In a one-dimensional model (corresponding to radial propagation, in the direction of the toroidal magnetic field gradient), power incident on a two-ion hybrid region can be reflected, transmitted, or absorbed (including mode conversion and subsequent damping of the electrostatic wave). If $R$ and $T$ are the power reflection and transmission coefficients for a wave, the solutions of the tunneling equation 36 for incidence from the high field side can be approximated as $R_{h}=0, T_{h}=\mathrm{e}^{-2 \eta}$, and for incidence from the low ficld side, $R_{l}=$ $\left(1-e^{-2 \eta}\right)^{2}, T_{1}=\mathrm{e}^{-2 \eta}$, where $\eta$ is referred to as the tunneling parameter. Waves can also be reflected at the fast wave cutoff layer $\left(n^{2}=S+D\right)$ near the plasma edge; we term this coefficient $R_{w}$. Note that in high power ICRF experiments, the wave absorption and its subsequent effect on the temperatures of the component species are of primary interest. Here, however, the quantities of interest are the wave fields themselves; we are not concerned with absorption but only transmission and reflection.

In the experiments described above, propagation incident from the low field side of the conversion laver led to large signal transmission (comparable to cavity mode levels in vacuum transmission), suggesting that $R_{l}$ was large (i.e., close to unity). 
Wave incidence from the high field side led to significant attenuation of the received signal. Because the transmitting and receiving probes were at approximately the same major radius. the observed at tenuation could result from two effects. The wave energy could be reduced by actual absorption in passing through the conversion and minority cyclotron layers. Attenuation could also occur if the wave energy that was transmitted through the layer from the high field side became trapped on the low field side before reaching the toroidal location of the receiving probe. due to large values of $R_{l}$ and $R_{w}$.

To simply the probiem further. we assume that $R_{w}=1$ (i.e., complete reflection at the fast wave cutoff) and that $\eta>1$ (i.e., $T_{h}$ and $T_{l}$ are small compared to one). We further ignore the phases of multiple reflections, which are wavelength dependent and will give rise to eigenmodes. Summing the contributions from multiple passes of a wave incident from the low field side (keeping terms to lowest order in $T_{l}$ and $T_{h}$ ). the power on the low field side is

$$
P_{l}=I_{0} \frac{\left(1-R_{l}-2 T_{l} T_{h}\right)}{\left(1-R_{l}\right)}
$$

where $I_{0}$ is the launched power. Similarly, the power on the high field side for a wave incident from that side is found to be

$$
P_{h}=I_{0}\left(1+\frac{2 T_{h} T_{l}}{\left(1-R_{l}\right)}\right)
$$

The ratio of the wave power on the high field side (with high field incidence) to that on the low field side (with low field incidence) is then just

$$
\frac{P_{h}}{P_{l}}=e^{-2 \pi}
$$

Hence, this rudimentary model suggests that the logarithm of the wave attenuation should scale as the tunneling parameter, $\eta$. The experimental data has been presented as the function $T$ (Eq. 3), which is proportional to the wave magnetic field and hence the square root of the wave power. Simple rearrangement yields $\eta=2.30 \Delta \log (T)$, where $\Delta \log (T)$ is the magnitude of the difference between the value of $\log (T)$ for low field and high field incidence on the conversion region. Note that in the actual 
experiments, the signals $P_{l}$ and $P_{h}$ are not measured for fixed resonance layer position. Rather, the probes are fixed and the resonance layer is moved radially past them by rarying the frequency. However. $P_{l}$ is relatively constant in the case of low field incidence as the resonance layer sweeps from a majur radius near the probe. to the inside plasma edge, and then out of the plasma. Assuming that $P_{l}$ is constant, $\Delta \log (T)$ is just the magnitude of the decrease in signal in the attenuation band measured with respect to the signal in the adjacent low-field incidence (i.e., higher frequency) region. For the examples shown in this paper. the attenuation depth is on the order of 0.6 - $1.5 \log$ unit, yielding estimates for $\eta$ of $1.4-3.6$. While a detailed comparison of these data to the predictions of current ICRF wave propagation codes is not within the scope of this paper, we note that the one-dimensional code CARDS 37 predicts values for the transmission cuefficient within the range estimated by the experimentally deterermined coefficient $\left(e^{-2 \eta}\right)$, given the uncertainties of launched wavenumber and minority species concentration.

\section{CONCLUSIONS}

A pair of small of magnetic probes, located well beyond the plasma limiter, have been used to launch and detect waves over a broad range of frequencies in TFTR. In the absence of plasma, the cavity mode cutoff of the toroidal vacuum vessel has been identified. With deuterium majority, hydrogen minority plasmas, the of transmission spectrum iricludes a distinct band of attenuation that occurs over frequencies for which the minority cyclotron layer position is located between the outer plasma edge (at $R_{p}+a_{p}$ ) and the approximate major radius of the probe itself (near $R_{p}$ ).

A simultaneous measurement of of wave transmission and spontaneous of emission by the plasma was made using synchronized, offset spectrum analyzers. The experiment showed that the low frequency rise in the transmission signal was caused by spontaneous emission and was not part of a second attenuation band. Fluctuations or variations in the spectra are probably due to wave modes because they are often 
coherent over a number of frequency sweeps. The modes are frequently large, particularly when acquiring spectra at rapid sweep rates. Spectra obtained by averaging successive, slow sweeps over long periods (on the order of seconds) may average out mode fluctuations if slow variation in some plasma parameter causes mode shifting comparable to the mode width.

Transmitting and receiving probes were both at nearly the same major radius and were close to the plasma center. and attenuation was observed only when the resonance laver, comprising a vertical cylinder, was in the outer half of the plasma $\left(R>R_{p r}\right)$. This result led to the prediction that, for the geometry of TFTR, attenuation bands from hydrogen, helium-3, and tritium minority species will all be distinct and resolved. Such bands were in fact found for helium-3 conditioning discharges (comprised largely of deuterium, hydrogen and helium -3), and for deuterium majority, hydrogen minority discharges into which a helium-3 gas puff was introduced. In the latter case, direct estimates of the helium-3 concentration could be made from the electron density increment.

The magnitude of the transverse wave polarization was measured during several discharges using the two orthogonal loop sensors that comprise each of probe. The spectra for each component were similar and $B_{\theta} / B_{\phi}$ varied from 0.2 to 1 .

A series of experiments with short helium-3 gas puffs were used to study the associated spectrum evolution. Since the signal to noise (or mode fluctuation) ratio decreased with rapid spectrum acquisition, successive spectra were averaged over various frequency windows. This technique vielded very clear data (Fig. 13) from which quantitative estimates were made. The characteristic time for the attenuation band to develop after a short gas puff was typically 60 - $100 \mathrm{msec}$, a time scale appropriate for penetration to the plasma core. In contrast, the electron density perturbation associated with the gas puff, integrated along a vertical chord near the plasma edge, reached a peak within $35 \mathrm{msec}$ and then decreased. This data suggests that the wave attenuation seen in the of transmission spectrum involves the plasma core: the wave is not a surface effect. 
The scaling of wave attenuation with the size of the helium-3 gas puff suggested an exponential dependence on the minority density: the data also allowed estimation of a detection limit of less than $0.25 \%$ for the fractional helium.3 ion concentration. I'sing the assumed exponential density dependence, appropriate averages were developed to compare the time evolution of the frequency averaged transmission spectrum directly with the spatially averaged (in terms of major radius) electron density signal from the interferometer. With a broad averaging window, good agreement was obtained.

By employing narrower frequency averaging windows (at the expense of increased signal fluctuation), some evidence for the dependence of the spectrum on major radius was obtained. In particular, the perturbation associated with a minority gas puff was slower to affect the higher frequency part of the attenuation band associated with smaller major radii (i.e., closer to the plasma core). The same overall ordering was found for the signals obtained from the density integral in Eq. (7), although the time dependence of that signal did not reveal an actual lag but only a decrease in slope for the signal associated with the averaging window at smaller major radii. It was speculated that the actual time lag observed in the evolution of the transmission spectrum could imply that the plasma core is weighted more heavily than would be indicated by Eq. ( 7 ); such an effect might arise from the peaked temperature profile.

Finally, a connection was made between the experimental observations and the 1. $\mathrm{d}$ theory that describes wave reflection, transmission, conversion and absorption near the two-ion hybrid layer resonance and cutoff in terms of exponential functions of a tunneling parameter, $\eta$. Although greatly oversimplifying the actual experimental situation, a simple relation was found between the depth of the minority attenuation band in the transmission spectrum and $\eta$; the experimentally derived values for the transmission factor are in the range predicted by other modeling codes. The unusual experimental geometry suggests that a global, 3-d modeling effort would be warranted to help understand the details of the spectral structure. Conversely, the clear experimental results present a new opportunity to demonstrate the validity of current modeling codes. 
The behavior of the spectral signals for large minority concentrations has not been investigated. If the two-ion hybrid resonance and culoff define the region of wave interaction. then the increasing displacement of the conversion region from the cyclotron laver will need to be considered. Certainly. at high enough minority levels. the observed attenuation band would be expected to exhibit a shift to lower frequency. We note also that only helium-3 perturbation experiments have been carried out thus far: puffing of hydrogen is infrequent in TFTR. Thus. the identity of the hydrogen attenuation band has been inferred rather than explicitly demonstrated.

Extension of this diagnostic technique to neutral beam or ICRF heated discharges will entail further development. During an ICRF pulse. of course, the fundamental heating frequency, and even its harmonics, are enomous compared to the signal levels detected in these experiments. However, even during beam injection, the spontaneous ion cyclotron emission that appears at harmonics of the deuterium cyclotron frequency at the outer plasma edge 26 is if comparable magnitude to the driven wave signals. In the ICRF case, no simple solution is in sight, but use of higher applied power and synchronous of modulation and detection may permit operation during heam pulses.

The conceptual simplicity of the measurements described here suggest diagnostic utility. In spite of the appearance of large modes, the signals derived from spectra that are frequency averaged over selected bands exhibit good signal to noise ratios and are sensitive to very small minority concentrations. By rewriting Eq. (7) as the appropriate weighted average in frequency, the signal could be used as a measure of the average total minority density within corresponding limits in major radius. In contrast to the interferometric electron density measurement, these spectra offer charge-to-mass ratio resolution for common light ion species. It seems likely that this diagnostic technique will be of use in measuring tritium ion concentrations during the upcoming DT experiments in TFTR. 


\section{ACKNOWLEDGEMENTS}

The author would like to thank C.K. Phillips, D. Manos, H. Park. A. Ramsey, $D$. Smithe and $K$. Young for useful discussions. The expertise of the diagmestics and operations staff of TFTR was of course essential to the successful completion of these experiments. The skilled work of . II. Candelori and H. Dymowski in support of the of probe diagnostic construction and installation is gratefully acknowledged. This work was supported by U.S. Department of Energy Contract No. DE-ACO2-76-CHO-30ri3. 


\section{REFERENCES}

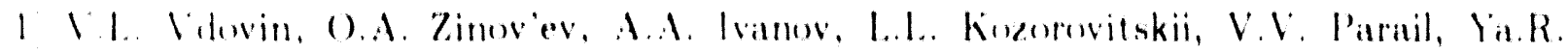

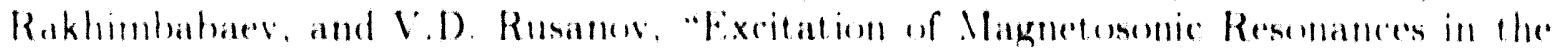

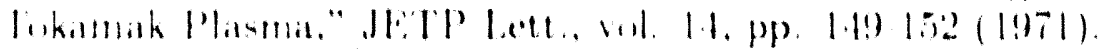

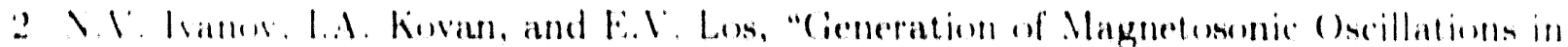
the linkimat IO 1," JE.TP Lett., wol, 14, pp. 1:38-1.10 (1971).

3 I. P. Bhatnagar, G. Bosia, M. Bures, F. Desoppere, A.Ml Messiaen, ('. Piret, (i. Celesca, P.F. Landenplas, and R.R. Weyants, in Procesdings of the Jome linenna. Cimoble lintomational Symposzum on Heating in Tomidal Plasmas. Cirenoble, 1978 (1'ergatment. ()xford, 1979), wol. 1, pp). 133-138.

1 1). Q. Hwang and R.W. Cimuld, Phys. Fluids, vol. 23, pp. 61.4-623, 19860.

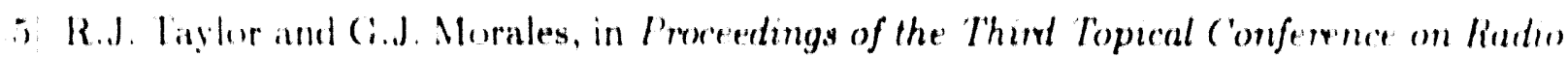
Fiequency Heating (California Institute of Technology, Pasadena, 1978), pl). (5.1 ( 53.3

6 T.H. Stix, "Fast Wave Henting of a T'wo-Component Plasma," Nuclear Fusion, vol. 15, pp. $737.75 .4(1975)$.

i P.I) ('oleman, "Propagation and Damping of the Fast Alfuen Wave in the lexas Tech linkamak," Ph.l). Thesis, Texas Terhnical University (Lubbock, Texas), lgxis.

* K.F. Lat. I.K. Man, B.1). Fried, and R.J. Taylor, "Energy Propagation and Field

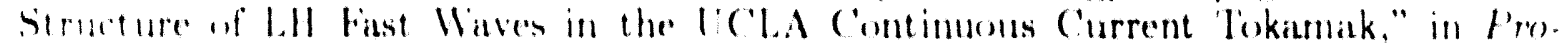

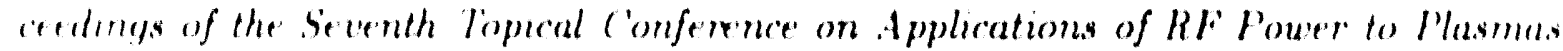
(Kissimmer, H. 1989), Mll, New York, pp. 111.11.1 (1987).

9) (i.J. (ireene. "ICKF ('oupling and Wave Propagation in a Tokamak Pasma," Ph.l). Thesis, Califormia lustitute of Terhomlogy (Pasalena, California), 198.1.

10) TFR (irmup, "Excitation and Damping of the Fast Magnetosonic Wave in TFR near the Harmonic ('yclotron Frequency," in Percedings of the Thind Intemational Mecteng on Theoretical and Expemmental Asperts of Heatung of Toroudal Plasmass, (Cirenoble. June 24 July 2, 1976) vol. 1, pp. X8. (9.1.

II Lguipe TFR, "Magnetosonic Wave (ieneration and Damping in the TFR Tokamak near the lon Cyclotron Frequencies," in Plasma Physacs and Controlled Vucle ar Fiusion heserareh 1976, Proceedings of the Sixth International Conference, Berchtesgaten. 11:1EA, Vienna, 1977), vol. 111, pp. 39-17.

12. J. Jacquinot, B.D. MeVey, and J.E. Srharer, "Wode Conversion of the Fast Magne usonic Wave in a Deuterium-Hydrogen Tokamak Plasma," Phys, Rev. Lett., vol. 39, pr. $\times 891(1977)$.

13 K.C: Budden, Kadoo Waves in the lonosphere, (Cambridge University Press, 1961).

1.t Y. Lapierre, "Magnetosonic wave propagation in the mode conversion regime," J. Plasma Physics, vol. 29, pp. 223-2.11 (1983).

15 S. lizuka, K. Odajima, H. Kimura, S. Sengoku, H. Matsumoto, T. Sugie, K. Taka 
hashi, I. Yamauchi, K. Kumagai, T. Kawakami. H. Takeuchi, T. Matsuda, K. Ohasa. .1. Nagami, S. Yamamoto, T. Nagashima. H. Maeda. and Y'. Shimomura, "Propagation and Absorption of the Fast Magnetosonic Nave in the lon-Cyclotron Range of Frequencies in the DIVA Tokamak." Phys. Rev. Lett., vol. 45, pp. 1256-1260) (1980).

16 Ci. G. Burg and R.C. C'ross, "Guided Propagation of Alfen and lon-lon Hebrid Waves in a Plasma with Two lon Species," Plasma Physics and Controlled Fisson, vol. 29. Pp. $681+896(1987)$.

17 M.J. Ballico and R.C. Cross, "Measurements of ICRF Edge Wave Fields in a Tokamak." Plasma Physics and Controlled Fusion, vol. 33, pp. 1841-1861 (1991).

18 R. Van Nieuwenhove et al., "Observation of a localised rf electric field structure in the scrapentf laver during ICRF on TEXTOR and ASDEX." 16th European (ion. ference on Controlled Fusion and Plasma Physics (Venice. Italy, 1989), Europhysics Conference Abstracts, vol. 13B, part 111, pp. 1065-1068.

19 G.J. Greene. "ICRF Antenna Coupling and Wave Propagation in the Caltech Research Tokamak." in Proceedings of the 1984 Intermational Conference on Plasma Physics. (Lausanne, Switzerland. 1984), vol. I, p. 63.

20) P. Lee. R.J. Taylor, W.A. Peebles, H. Park, C.X. Yu, Y. Xu, N.C. Luhmann. Jr., and S.X. Jin, "Observation of Mode-Converted Ion Bernstein Wuves in the Mircotur Tokamak," Phys. Rev. Lett., vol. 49, pp. 205-208 (1982).

21 TFR Ciroup, A. Truc, and D. Ciresillon, "Observation of Fast and Slow Modes A. sociated with ICRF Heating in the TFR Tokamak by $\mathrm{CO}_{2}$ Laser Scattering," Nucl. Fusion, vol. 22, pp. 1577.1587 (1982).

22 H. Park. W'.A. Peebles, N.C. Luhmann, Jr., R. Kirkwood and R.J. Taylor, 'Obser. vation of the Externally Excited Fast Magnetosonic Wave via Collective Thomson Scattering.' Plasma Physics and Controlled Fusion, vol. 27, pp. 1195-1202 (1985).

23 R. Mc Williams, ed. Radio-Frequency Pouer in Plasmas: Eighth Topical Conference (Irine). (AIP. New York, 1989).

21 D.G. Swanson, "Radio frequency heating in the ion-cyclotron range of frequencies," Physs. Fluids, wol. 28, pp. 2645-2678 (1985).

25 D.X. Smithe, P.L. Colestock, R.J. Kashuba, T. Kammash, "An Algorithm for the Calculation of Three-Dimensional ICRF Fields in Tokamak Geometry," Nucl. Fusion. vol. 27. pp. 1319-1340)(1987).

26 G.J. (ireene, "Neasurement of Ion Cyclotron Emission and ICRF-Driven Waves in TFTR," in Proc. 17th European Conference on Controlled Fusion and Plasma Heating (Amsterdam, 1990), vol. 14B, part IV, pp. 1540-1543.

27 G.J. Cireene, "Development of a metallic shield for the 11 iR of probe diagnostic," Rev. Sci. Instrum., vol. 61, pp. 3028-3030 (1990).

28 P.L. Colestock, private communication.

29 F. Cap, "Some Remarks on Toroidal Problems," Beitrage aus Plasma Physik, vol. 18, pp. $207-216$ (1978). 
30 F. Cap and R. Dentsch, "Toroidal Resonaturs for Electromagnetic Waves," IEEE: Transactions on Microwave Theory and Techniques, vol. WTT 26, pp. 478 +86) (1978).

31 G.J Girecte. "High Frequency Emission from lF IR Plasmas," in Fres. 15th European

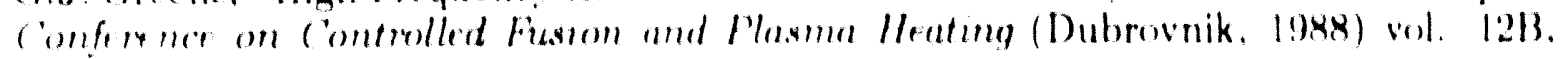
part 1. Pl. 107-1111.

32 F. II Terkins. "Heating lokamaks via the lon (yelotron and lon-Lon Hybrid Rese. nances." Nuel. Fusion, vol. 17. pp. 1197.122.1 (1977).

3:3 l' Stix. The Theory of Plasmu Hares, (McCiraw Hill, New York, 1962).

3. F.J. Sinakowski, B.C. Stratton, P.C. Efthimion, R.J. Fonck, R.A. Hulse, D.W. John wn. D.K. Mansfield. H. Park, S.D. Scott, and G. Taylor, "Measurements of Radial Protiles of He. "Transport Cuefticients on the TFTR Tokamak," Phys. Rev. Letters. vol. 6i), pp. 2255-2258 (1990).

35. D.K Mansfield, H.K. Park, L.C. Johnson, H.M. Anderson, R. Chouinard, V.S. Foote. C.H. Ma, and B.J. Clifton, "Multichannel far-infrared laser interferometer for electron density measurements on the tokamak fusion test reactor," Applied Optirs, vol. 26. pp. $4.69-47.4(1987)$.

36 D.G. Swanson. Plasma Wiales, (Academic Press, San Diego, 1989).

37 C.K. Phillips private communication. 


\section{FIGURE CAP'TIONS}

Fig (1) Dingram of the of probe wave propagation experiment.

Fig. (2) lransmioninn spectrum obtained with empty trikamak vessel (no plasma) Onset of cavity modes is apporent at $=73.111 / 2$.

fig. (3) Vurmalized transmission spect rum for deuterium majority hydrogen minor. it! whinic plasma ( $T$ is defined in text). Vertical dashed lines are plutted at frequencies for which the hydrogen cyclotron layer is at the outer plasma edge $(A)$ at the major radius of the probe $(B)$, and at the inner plasma edge (C).

Fig. (1) Simultaneous measurement of transmilted spectrum (A), spont aneous plasma of emisoin (B), and system noise level (C). Low frequency rise in transmission spectrum is due to spontaneous emission.

(iig. (5) Transmission spectra in deuterium majority, hydrogen minority plasmas for high and low toroidal field inlues. The three dashed lines plotted in each graph are as described for Fin. 3. (a). $B_{T F}=4.45 \mathrm{~T}$. (b) $B_{T F}=2.0 \mathrm{~T}$.

Fig. (6) Cold plasma dispersion surfaces for the discharge associated with Fig. 3, with $n_{H} n_{l}=0.05$ and $k=7 \mathrm{~m}^{-1}$. Rectangles at the top and bottom of the figure indicate the polotidal positions of the transmitting and receivite probes. lines are plotted at the minority cyclotron resonance position (A), the two ion hybrid layer cutoff (B) and resonance (C), and at the fast wave cutoff (D) and plasma boundary $(\mathrm{f})$ (a). $f=50 \mathrm{MHz}$ corresponding to high field incidence on the resondace laver (b) $f$ 65. $.11 \mathrm{~Hz}$, corresponding to low field incidence.

Fig. (i) Transmiasion spectrum for helium 'conditioning' discharge. Species mixture includes deuterium, hydrogen, and helium-3. Vertical dashed lines are plotted at frequencies for which the helium-3 cyclotron resonunce layer is at the outer plasma edge (A) and at the major radius of the probe location (B), and at frequencies for which the hydrogen cyclotron resonance laver is at the outer plasma edge (C) and at the major radius of the probe location (D)).

Fig. (8) Cold plasma dispersion surfaces for the discharge associated with Fig. 7 , with $n_{H} \quad n_{l}, n_{H e}, 0.05$ and $k=0.7 \mathrm{~m}$. Here lines are plotted at the hydrogen cyclotron resonance $(A)$, two-ion hybrid cutoff $(B)$, and resollance (C) layers; and at the helium 3 ryclotron resonance (D), two-ion hybrid cutoff (E). and resonance $(F)$ layers. Curve $G$ is plotted for the fast wave cutoff, and curve $\mathrm{H}$, for the plasma boundary. For the frequency chosen (50 $\mathrm{M} / \mathrm{H}$ ). both minority mode conversion regions are within the plasma.

Fig. (9) - Transmission spectra in a helium-3 conditioning discharge for orthogonal, transverse polarizations of the wave field. Vertical dashed lines are plotted as in Fig. 7 . (a). $B_{\phi}$ signal. (b). $B_{\theta}$ signal.

Fig. (10) - Vertical chord integrated electron density waveform from the interferometer channel at $R=2.68 \mathrm{~m}$. Rectangular box denotes period of helium-3 gas puff. Vertical dashed lines indicate time intervals for spectrum acquisition before (A) and after (B) gas puff. 
Fig. (11) Transmission spectra obtained from the two time windows indicated in Fig. 10. Vertical dashed lines are plofted as in Fig. 7. (a). Spectrum before gas puff. (b) Spectrum after helium 3 gas pulf.

Fig 112 Thansmission spect rum taken 200 msec after helium 3 gas puff using fast aweep -peed. Rectangles on frequency axis indicale averaging wimdows discussed in

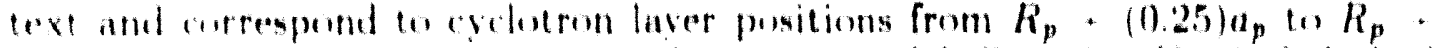
(1) ija, fir each minority species (hydrogen and helium 3). Vertical dashed lines are photted as in Fix. 7 .

Fig. (13) Time ewolution of spectra. averaged over frequency windows indicated in Fix. (12). Curve I Helium-3 window, small gas puff $(0.75 \mathrm{TL} / \mathrm{s})$. Curve B Helium 3 window, large gas pulf $(1.5 \mathrm{TL} / \mathrm{s})$. Curve $\mathrm{C}$ hydrogen window. large gas puff. Rectangle of time axis indicates duration of gas puff. Lines are drawn between experimential data points indicated by the symbols.

Fig. (1.4) Comparison of time evolution of helium.3 attenuation band with electron density perturbation near plasma edge during a helium.3 gas pulf. Curve A evolution of transmission spect rum $(\log (T)))$, averaged over same frequency window indicated for helium 3 in Fig. 12. Curve B - Chord integrated elect ron density increment from gas pulf $\left(\Delta n_{e} l(R)\right)$, with axis inverted and offset as shown for comparison with transminsion signal. Chord located at $R=3.17 \mathrm{~m}$. near outer plasma edge. limes are drawn between experimental data points indicated by the symbols.

Fig (15) Pho of Eq. (7) for experimental data during a helium-3 gas puff. Brond averaging withdow extended from $h_{p} \cdot(0.12) a_{p}$ to $R_{p} \cdot(0.62) a_{p}$. Circles are plotted for the areraged transmission spectrum indicated by the left side of the equation (Ing(t) i: triangles are plested for the right hand side of the equation (i) Ds), which contains the density integral. The value of $(i$ was fixed at ti. 10 in urder 1, match the pre puff values of the waveforms; the best fit for the data then required 11.1 .67 * 11) $20 \mathrm{~m}$. Rectangular box on time axis indicates duration of gas puff (1,5 Tl, s).

Fig (16) Evaluation of components of Eq. (7) for experimental data during a helium 3 gas puff. using two narrower helium-3 averaging windows. Circles are plotted for an averaging window from $h_{p}$ to $h_{p}$, (0.2) $a_{p}$; triangles are plutted for an averaging window from $R_{p} \cdot(0.5) u_{p} \omega R_{p} \cdot(0.7) a_{p}$. (a). Transmission signal $(\log (T))$. (b). Density integral $(\xi)$. 


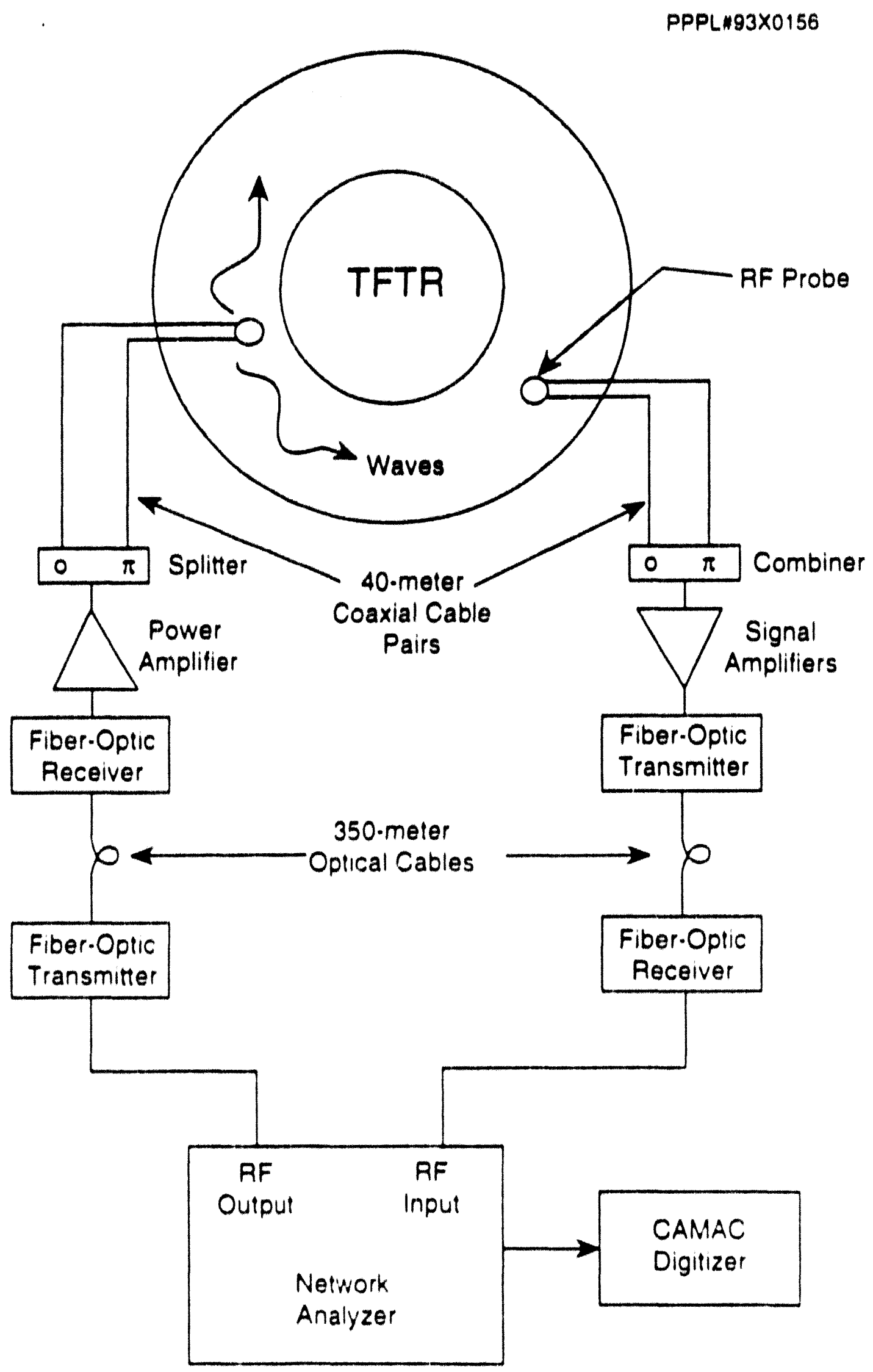




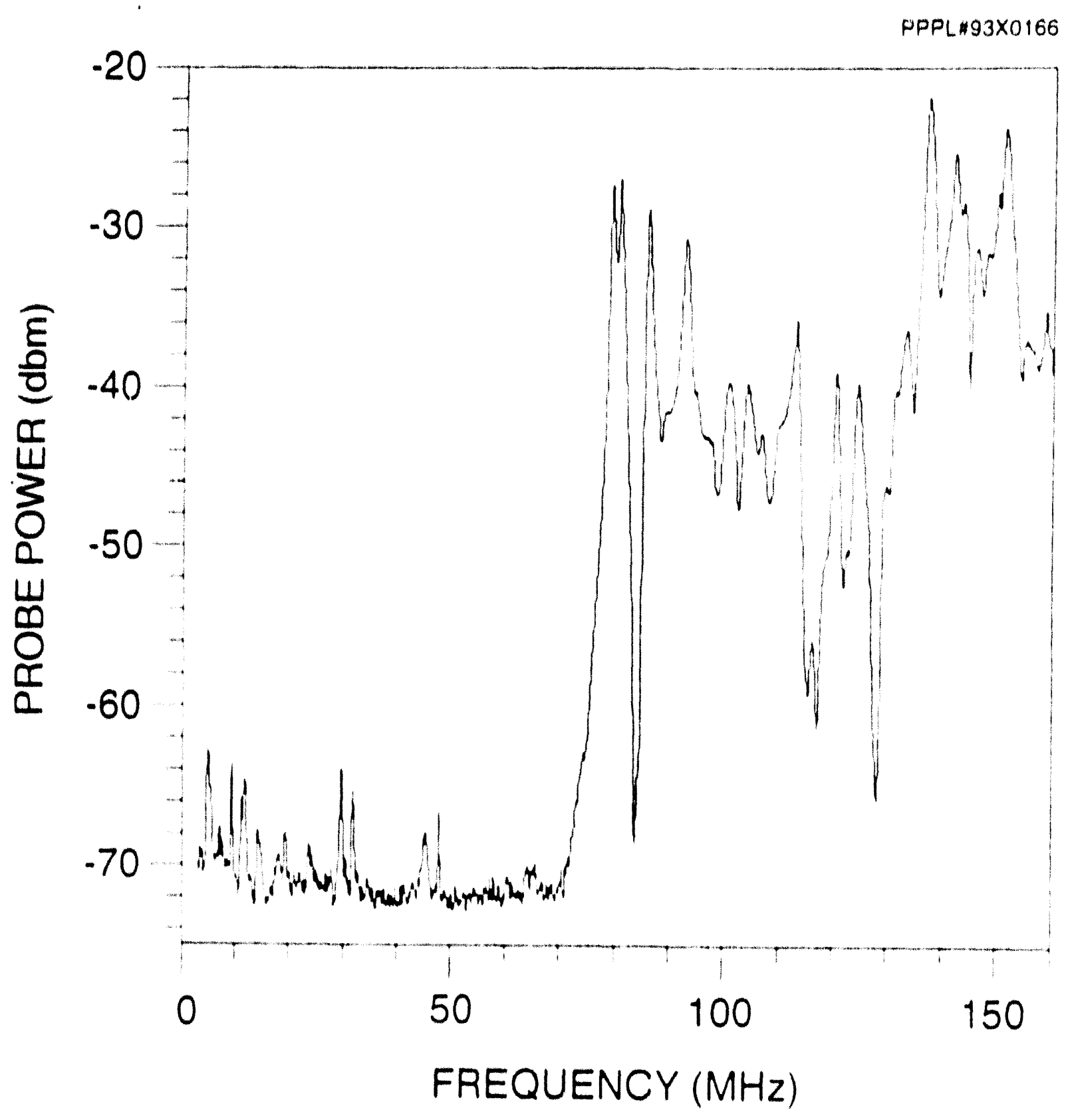

Fa. 


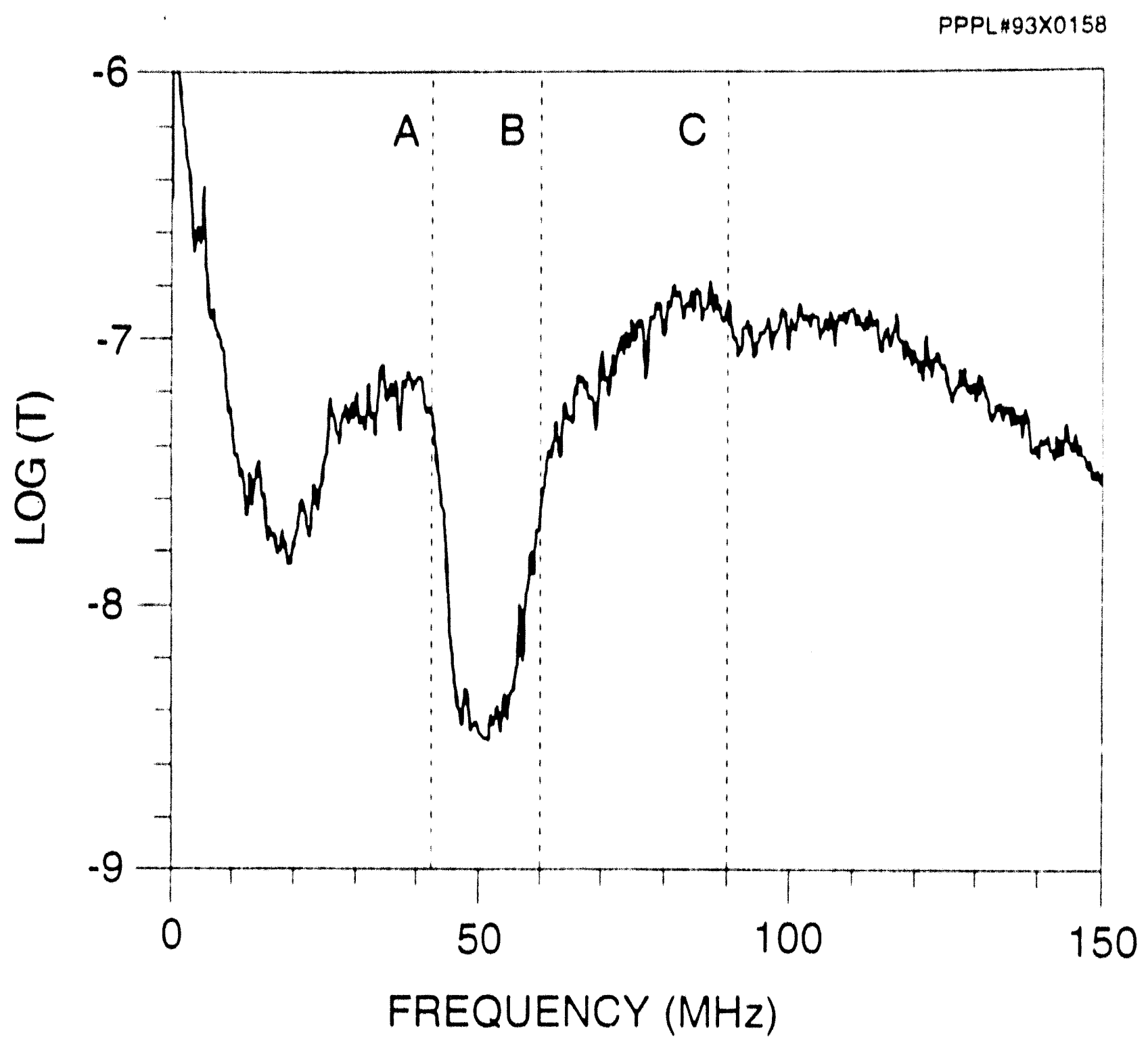




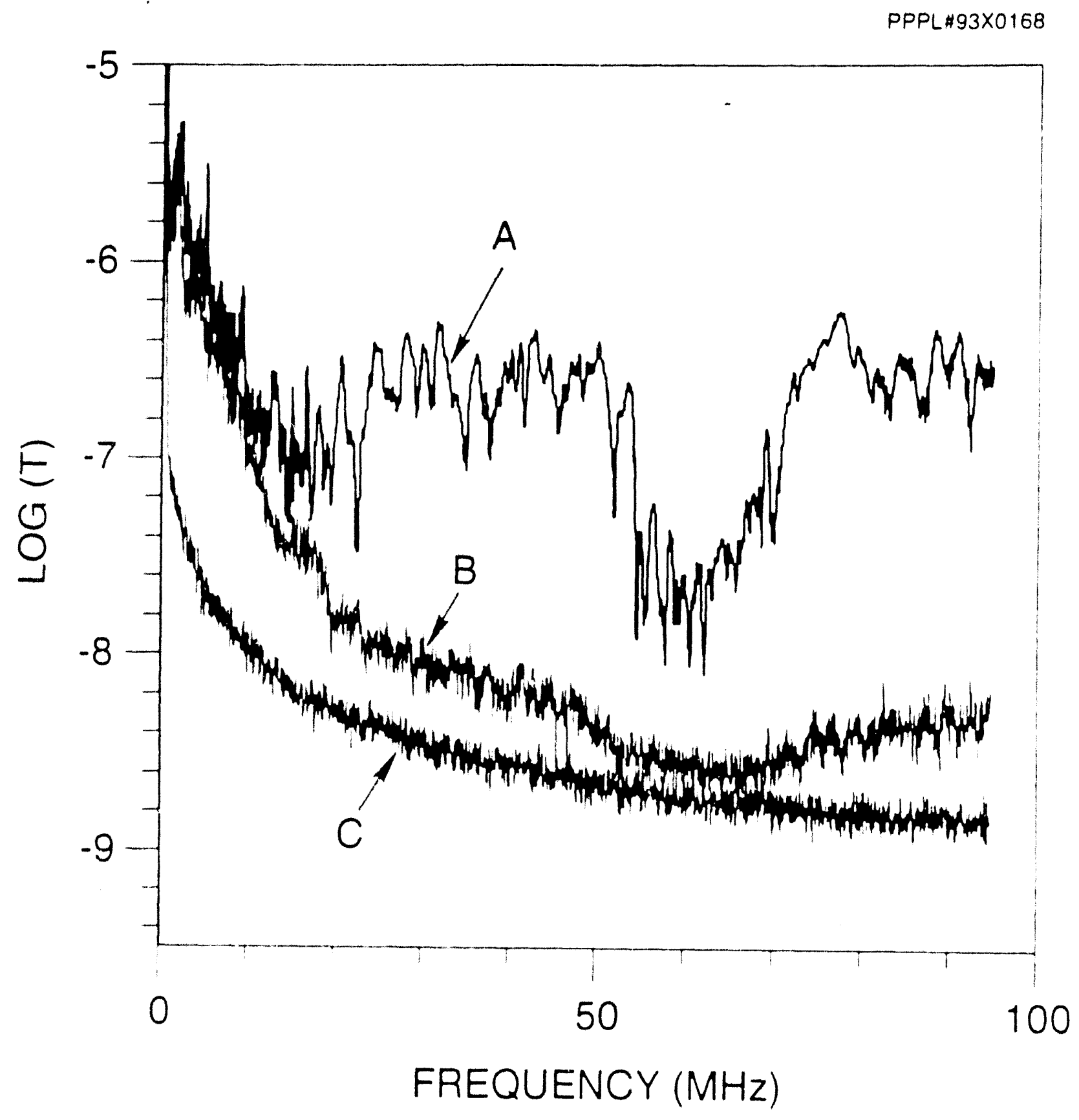

F.9. 4 

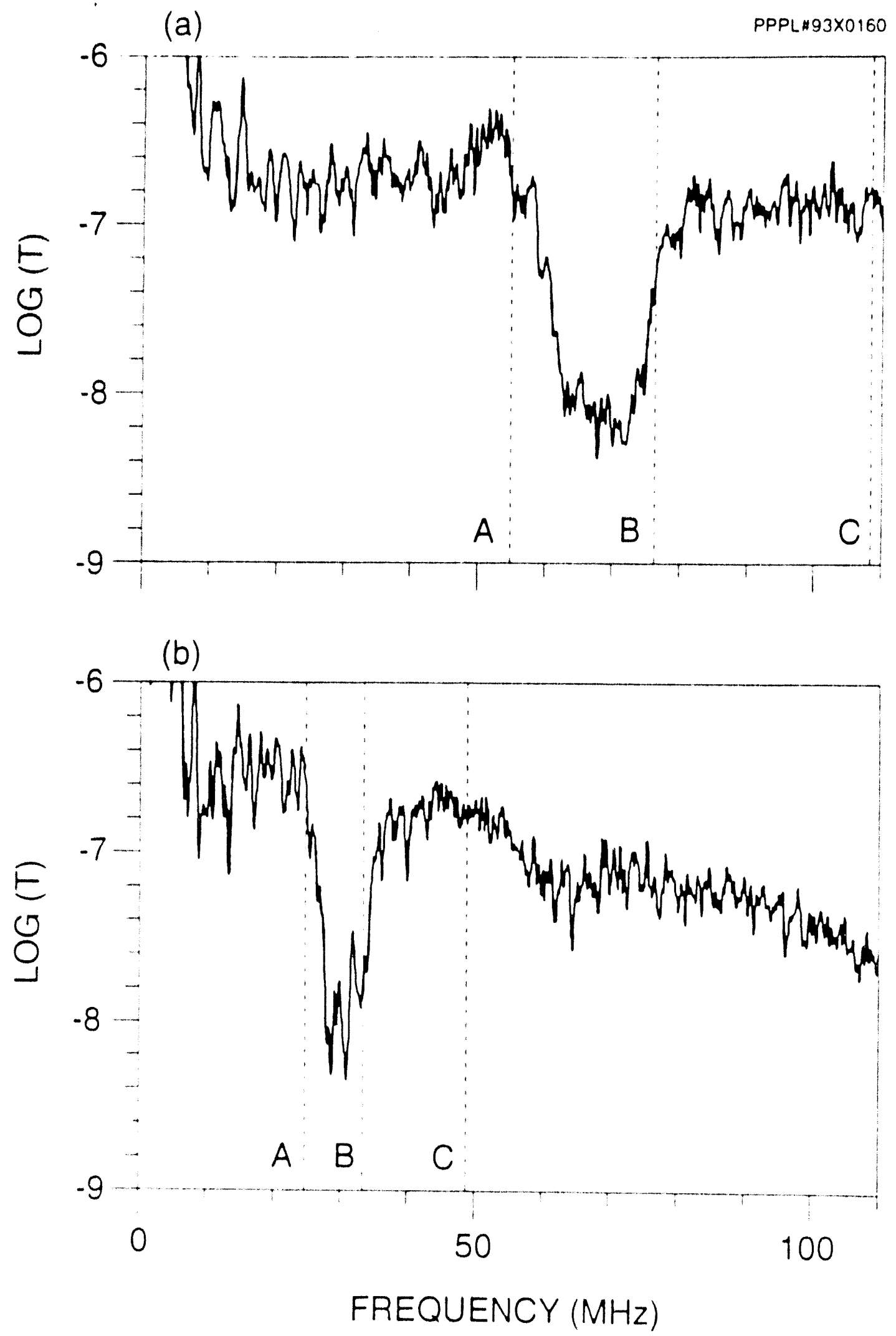


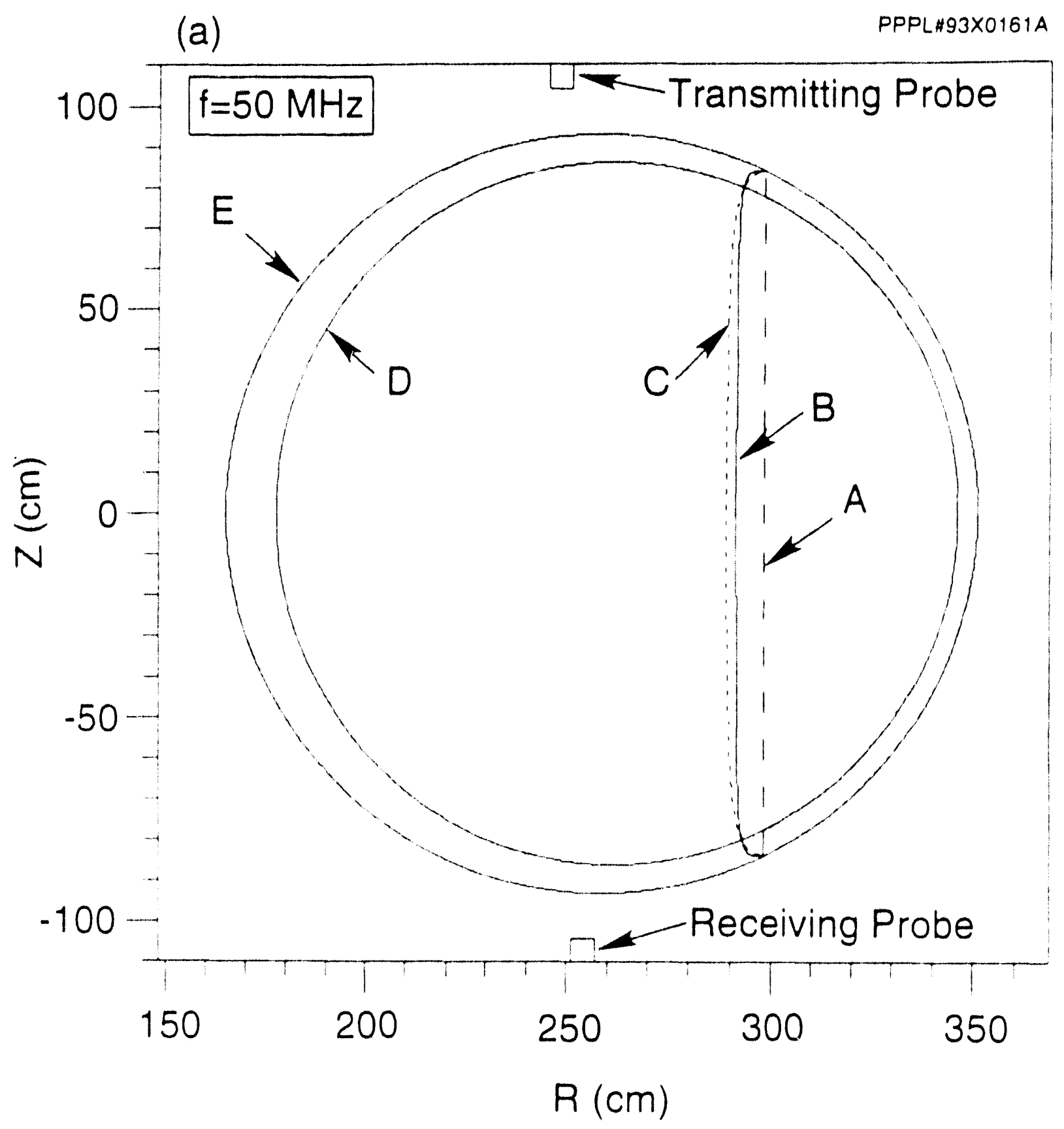

Elg. 6a 
(b)

PPPL\#93X01618

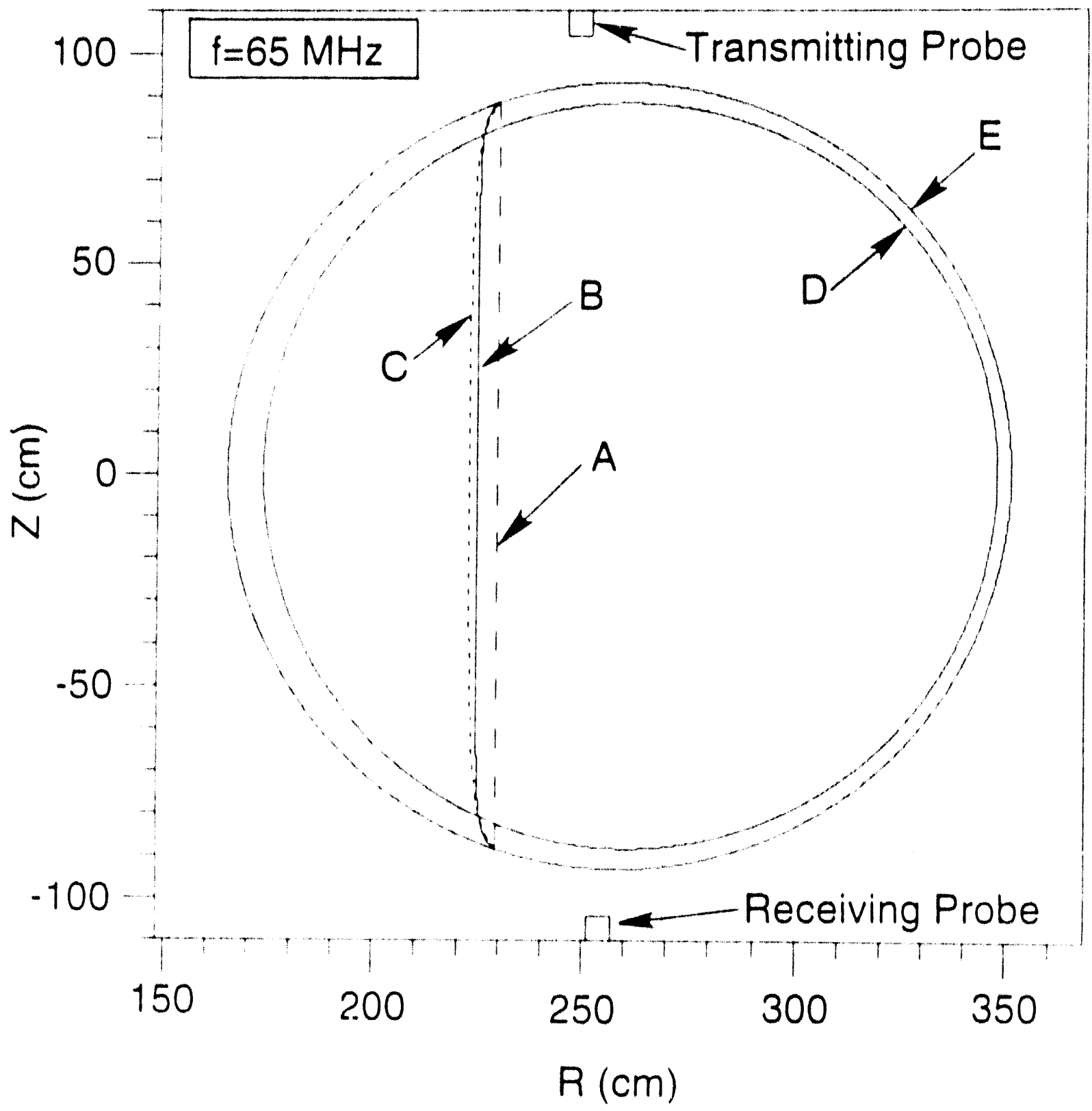

rig, 


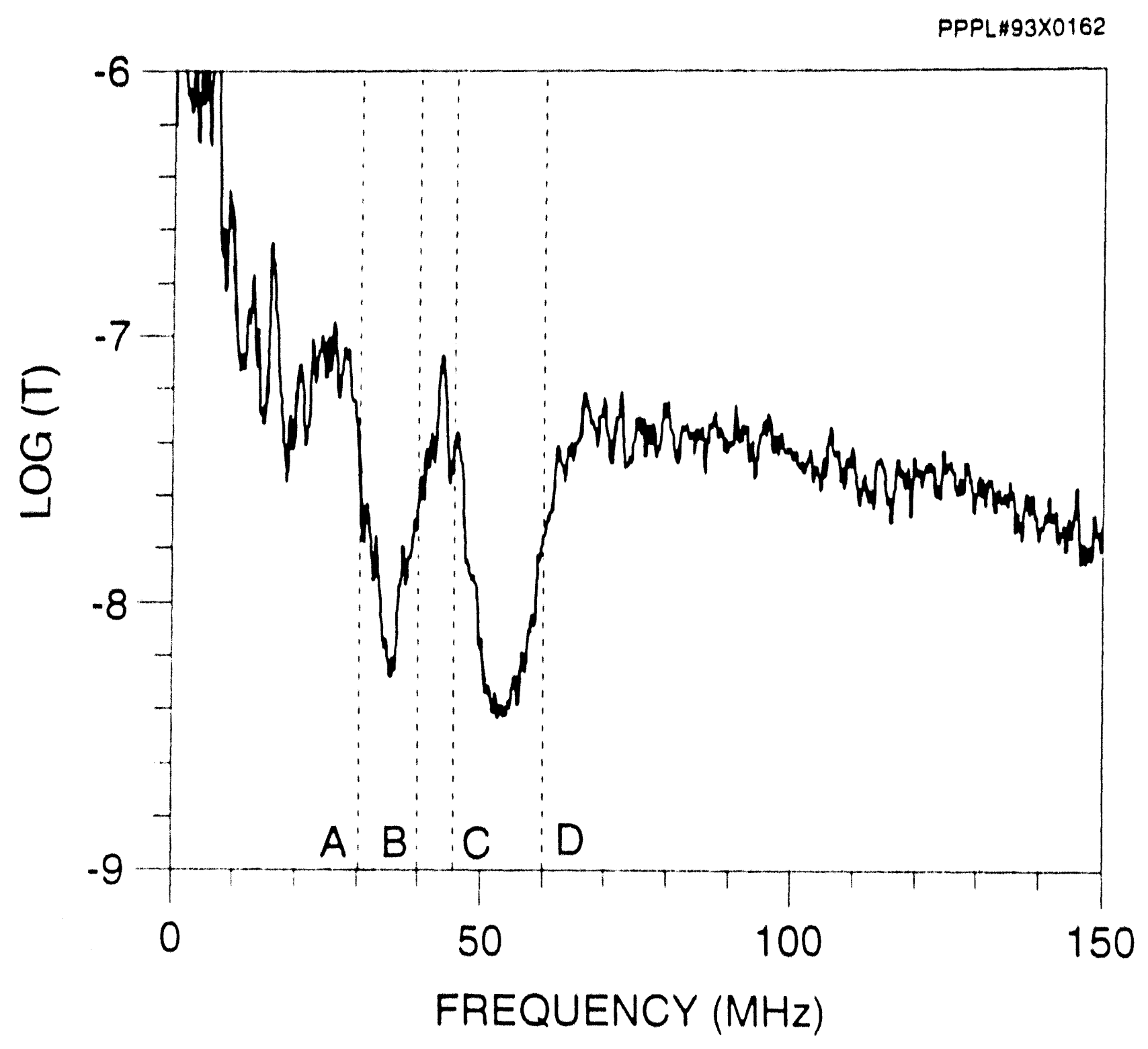




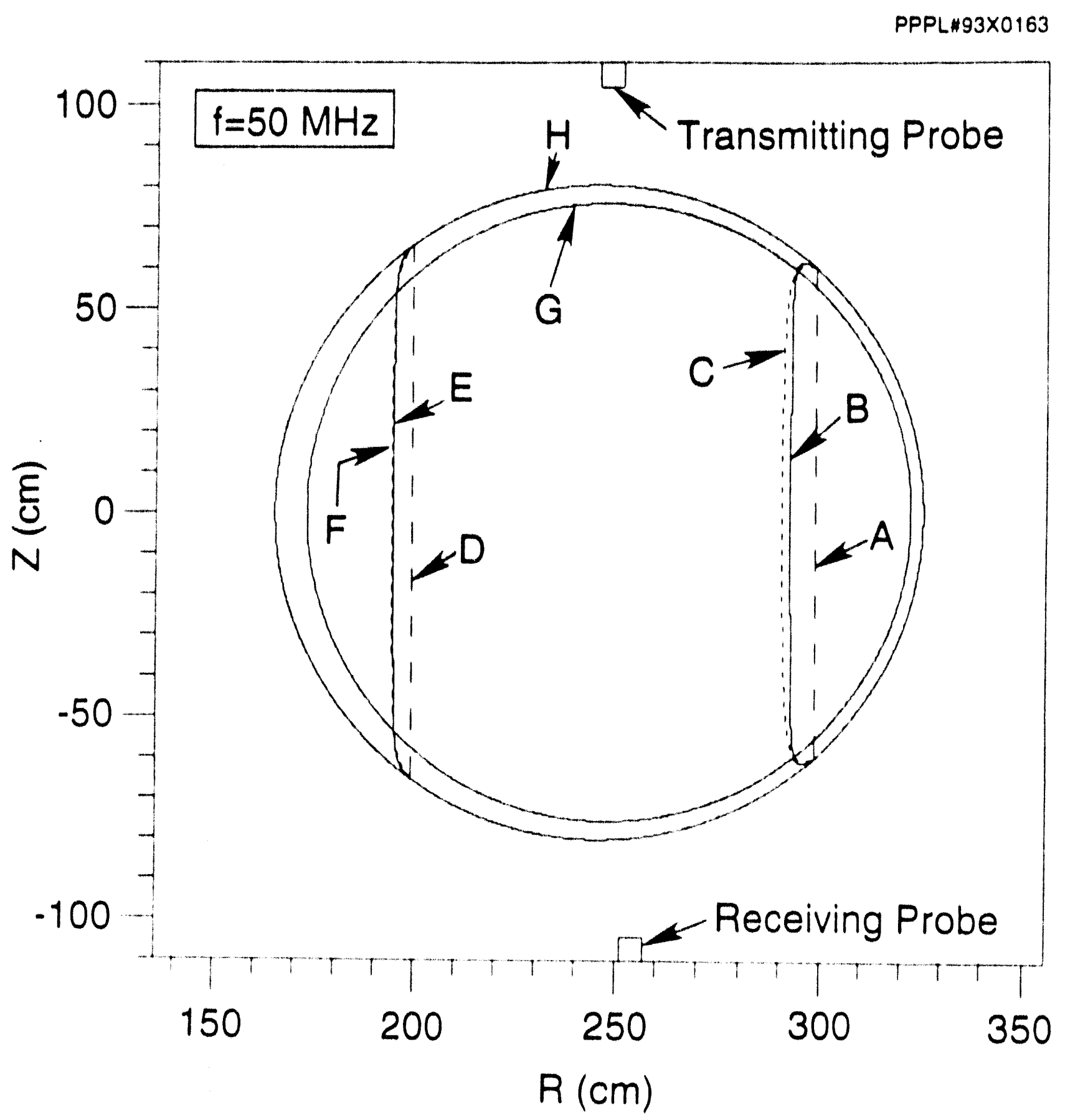



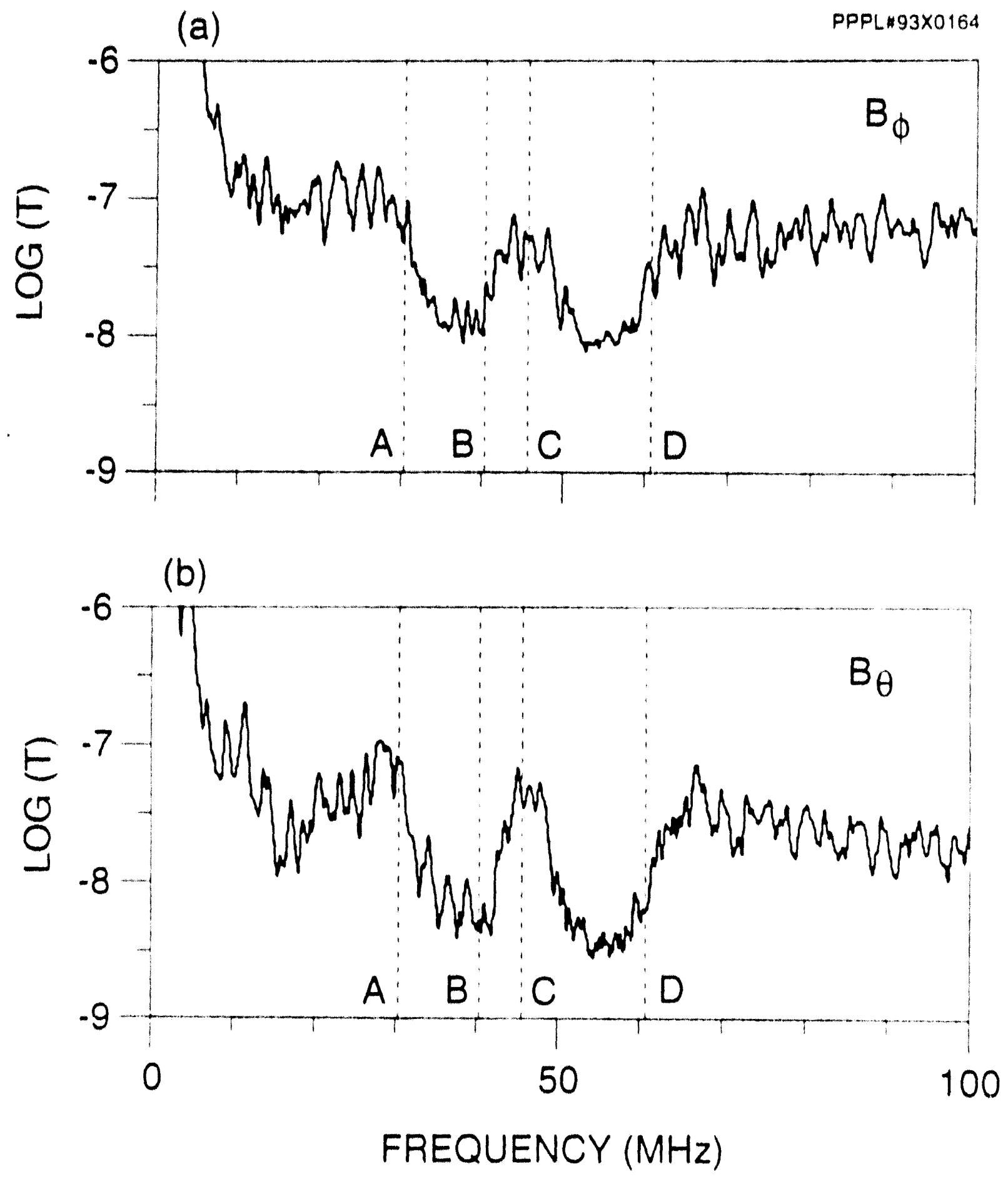


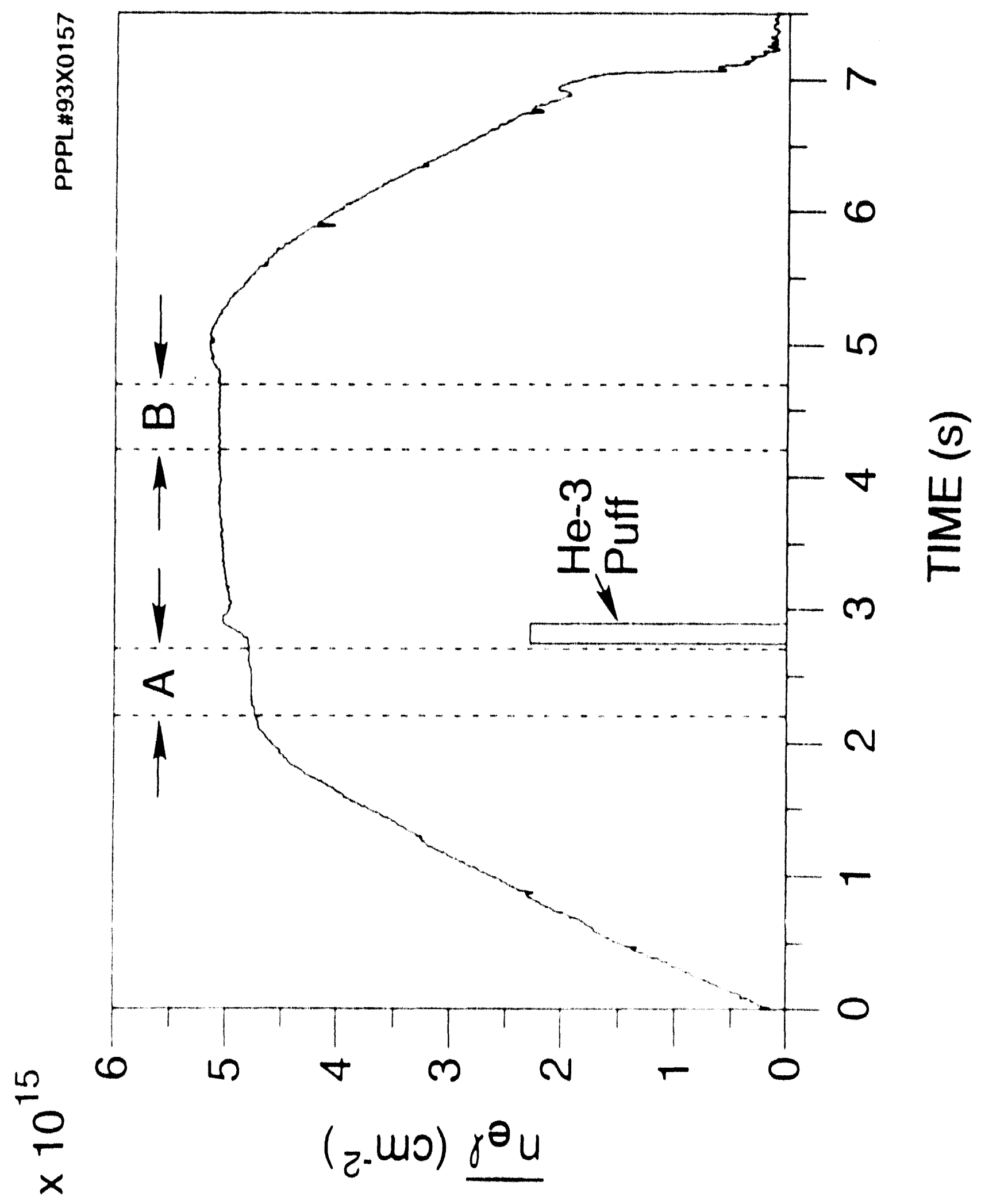



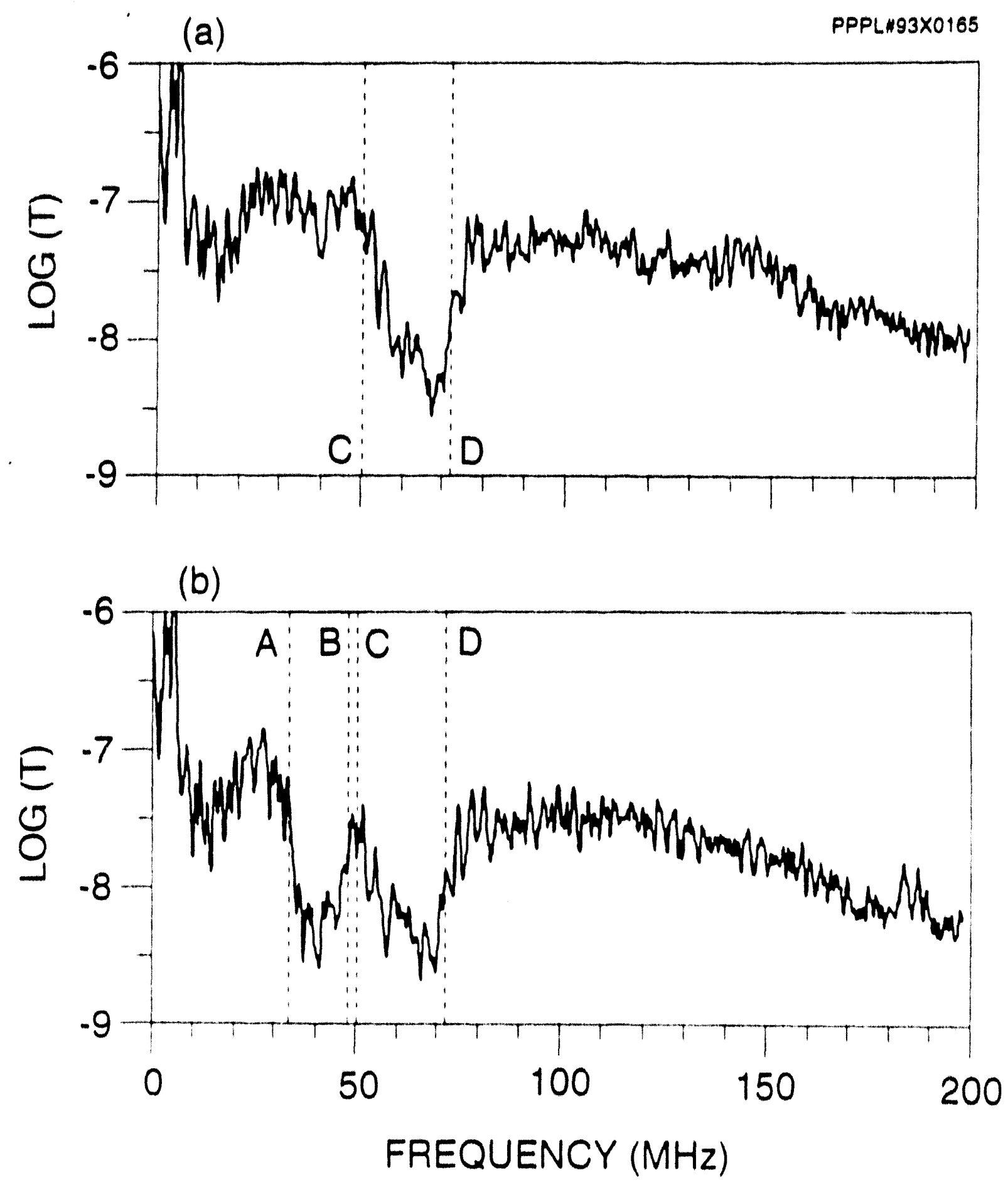

Fig. 11 


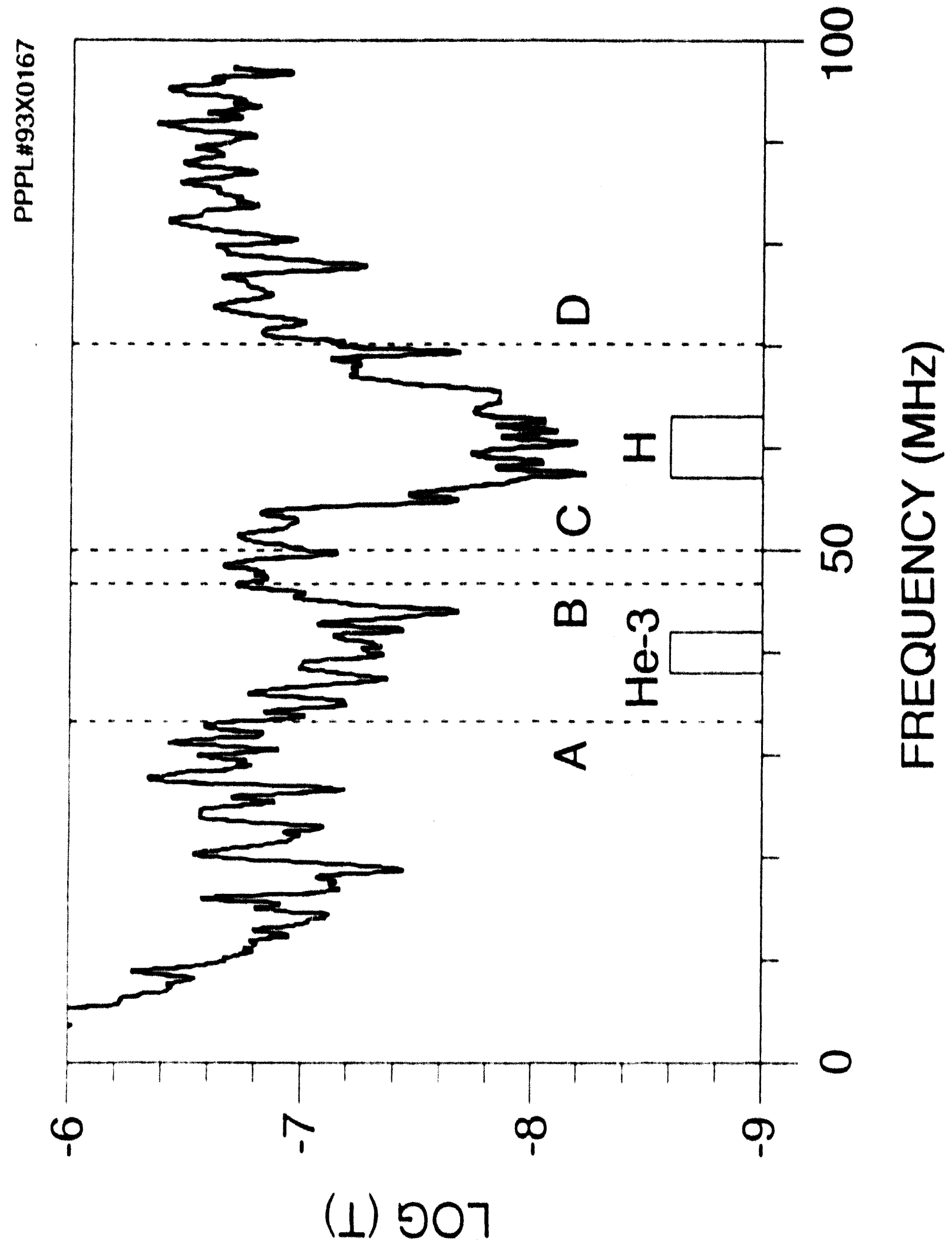




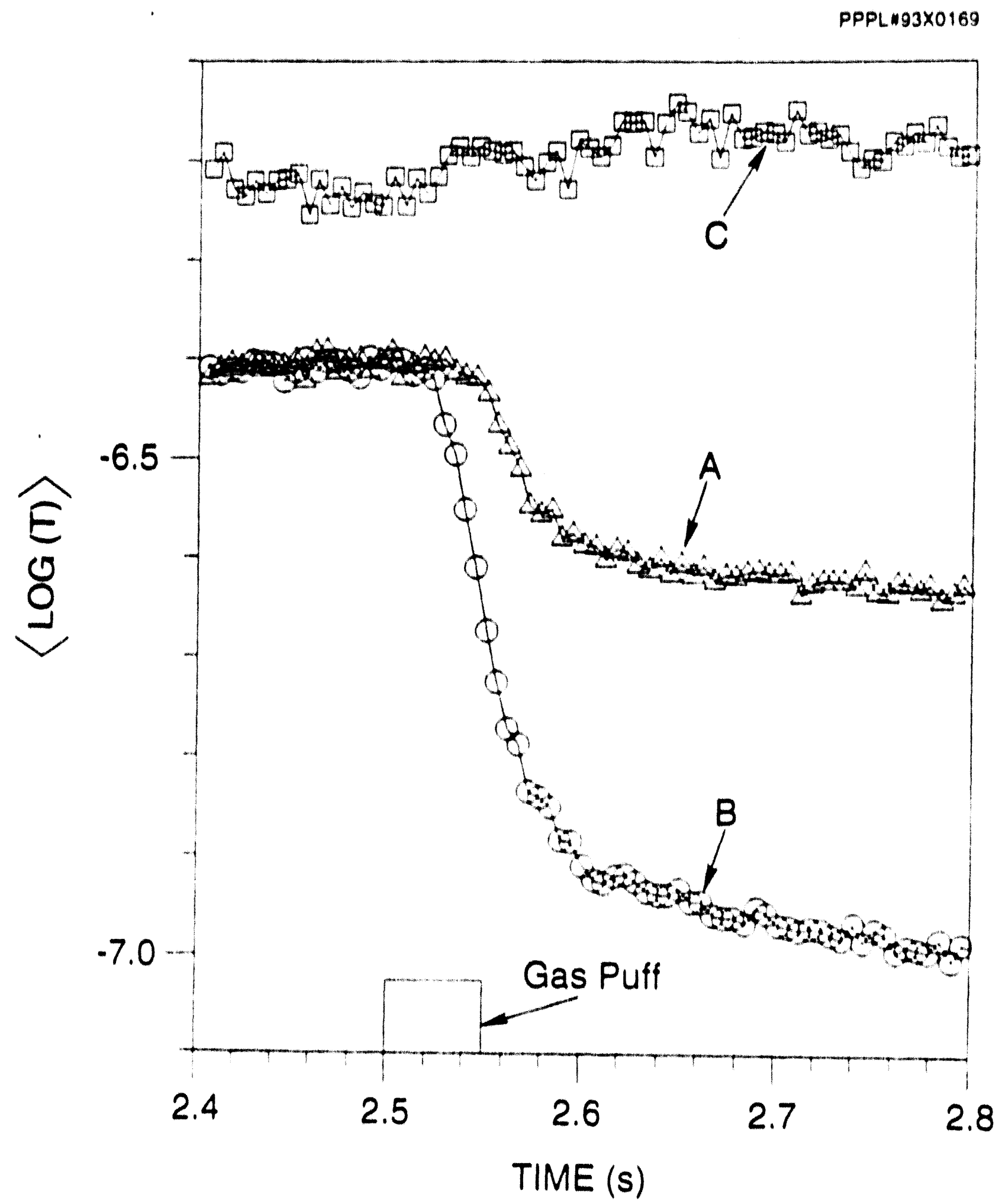




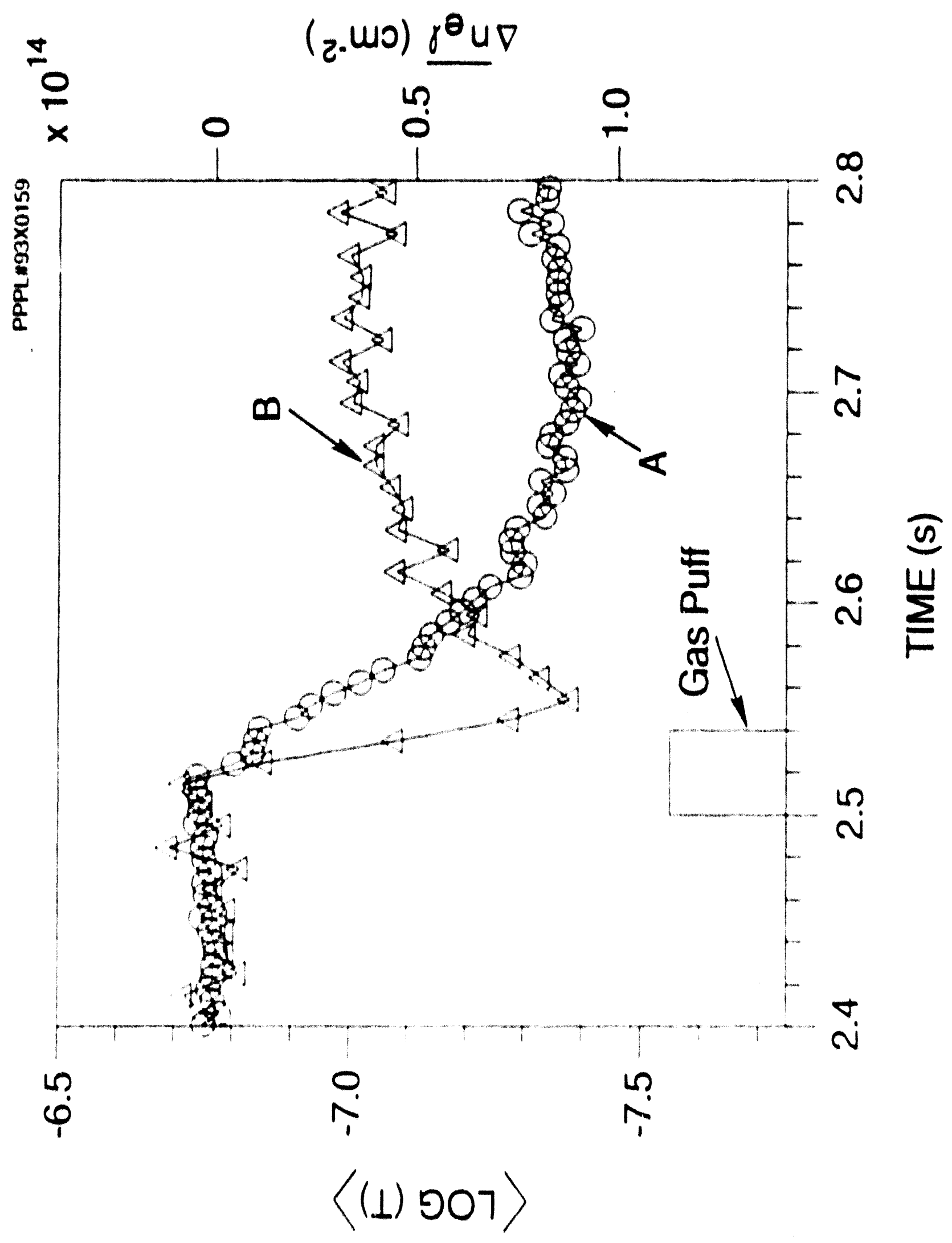




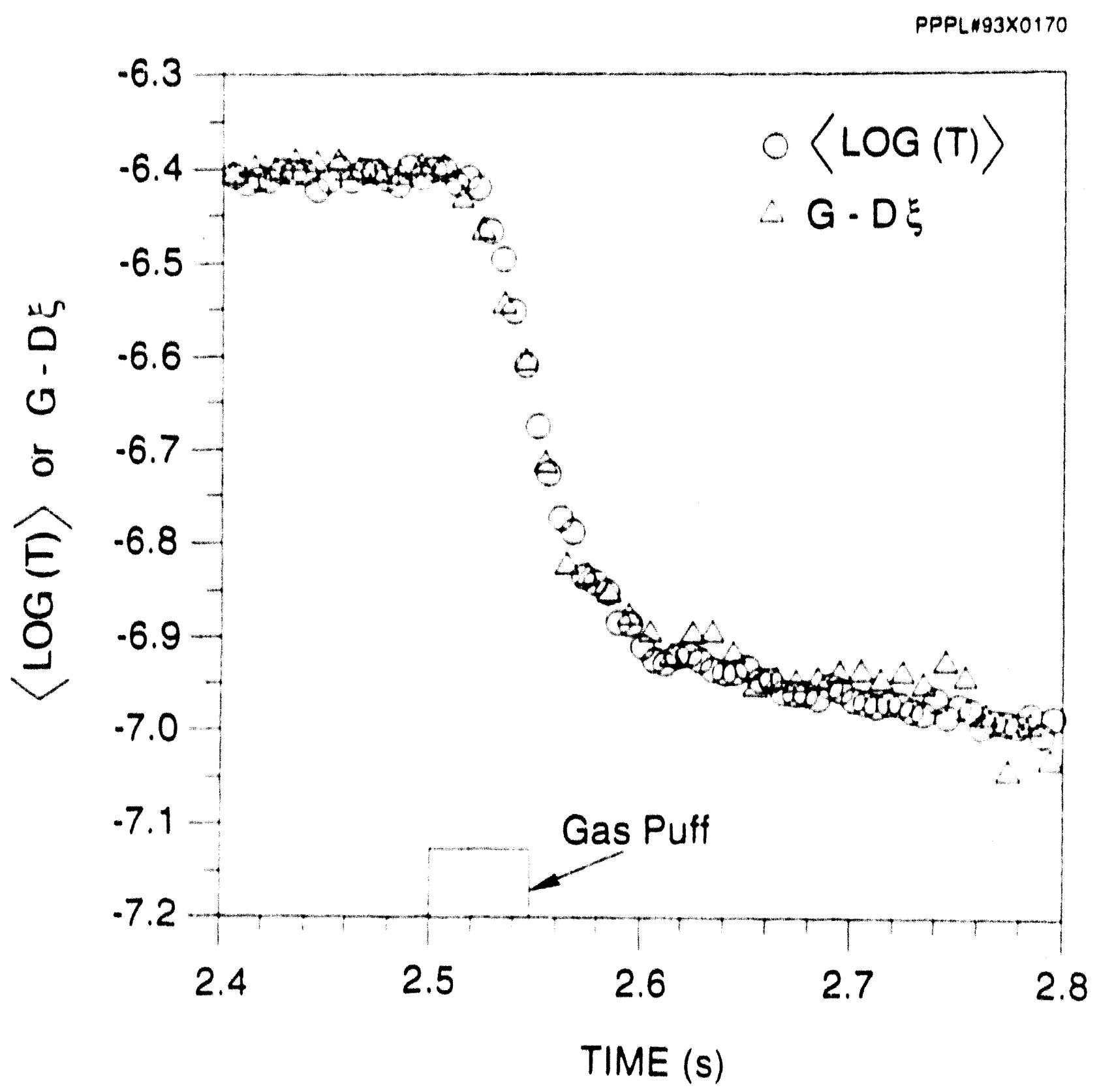



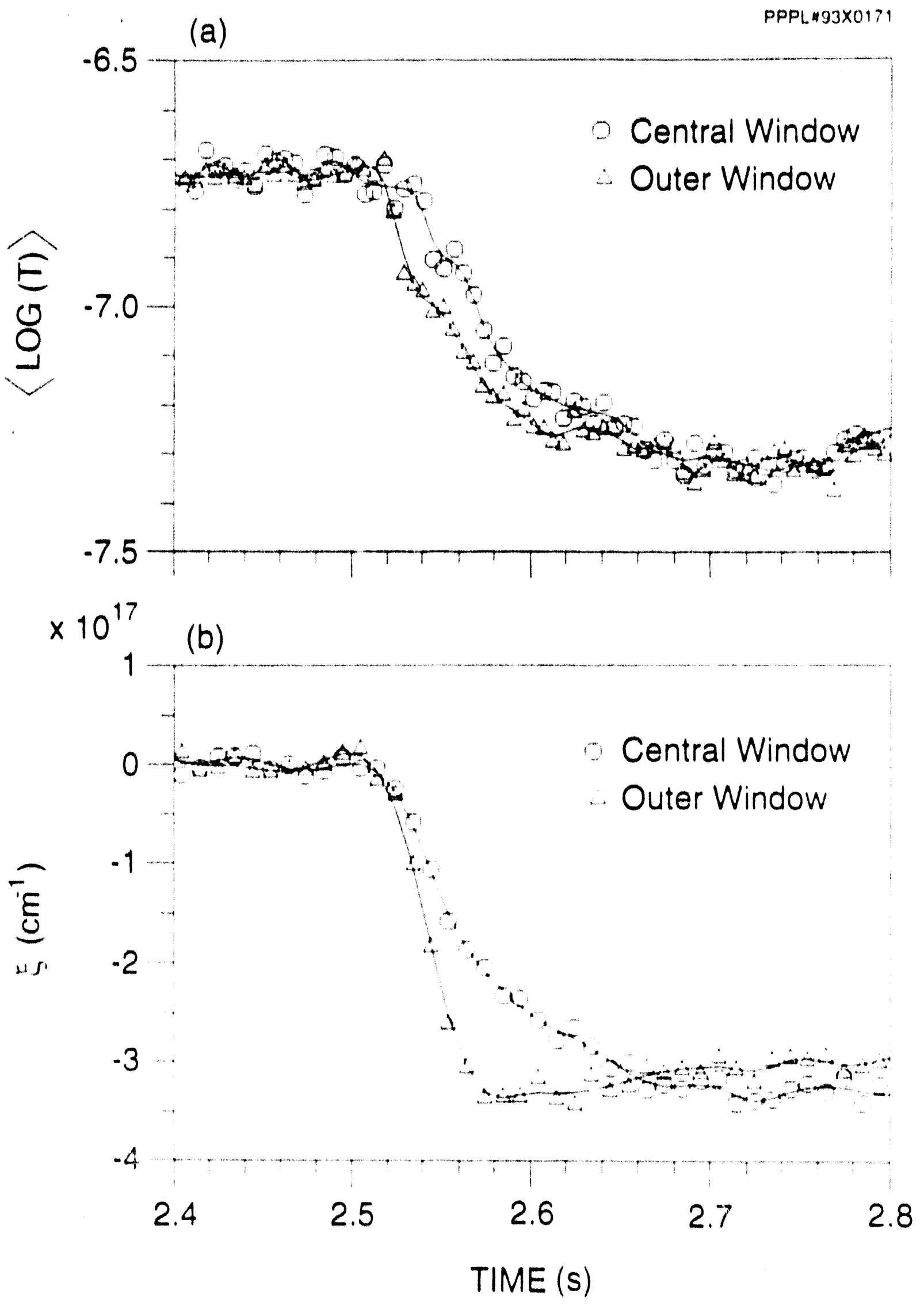
Or F Paolons. UnN of Wottongong. AUSTRALIA

Prol MH Bronnan. Univ of Sydnoy, AUSTRALIA

Plasma Research Lab Austaian Nat Univ, AUSTRALIA

Prol iR Jones Flinders Univ. AUSTRaLIA

Prot F Cap inst tor Theorebcal Physics. AUSTRIA

Prol M Hender. Insuout tor Theoreusche Physik AUSTRIA

Prot $M$ Coossens. Astronomisch Insourur BELGIUM

Ecole Royde Minturo, Lo do Phy Plesmas, BELGIUM

Commission Europen. OG XII-Fusion Prog. BELGIUM

Prof R Bowade Ryksumberwan Gent, BELGIUM

Or PH Sakenake Insourto Frica, BRUZIL

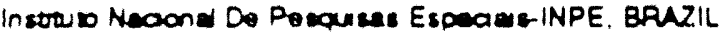

Doument Otho Alomic Enorgy of Cenade LU, CANADA

Dr MP Bectrynsk. MPB Tectinotogess. Inc. CANADA

Dr HM Skarsoard. Unv of Sackatchewen, CANADA

Prot J Tordmam. Unn of Montreal. CANAOA

Pof SR Sroenvasan. Umy ol Cajgary, CANADA

Prol TW Jomston, INRS-Energie, CANADA

Dr a Botion. Contro canadien de husion magnabour. CANADA

Or CR jemer. UnY of NDerm. CANADA

or P Lukac, Komensketho Unversata, CZECHOSLOVAKIA

Tho Lbranen. Cuham Leborabry. ENGLAND

Lorary. R61, Ruthertiond Apploton Laboralory. ENGLAND

Nhs SA Hutcrinson, JET Loray. ENGLAND

Dr SC Sherr UnN of South Profic. FIJI ISLANDS

$P$ suthonen. Univ of Hoisintw. FINLAND

Prot NN Buswac. Ecote Polytochnoure,. FRANCE

C Moutret Lo do Physique der Minux bonises. FPANCE

¿ Rad CENCADARACHE - Bal 506, F P ANCE

Prol E Economou Univ al Croto. GREECE

Ms C Runn UnN of loemna GREECE

De T Mud Acadomy Bbwographuc Ser, HONG KONG

Propont Lorary. Hunganan Acadomy of Sa. HUNGARY

or a Cas Gupta Sana inst of Nuciaar Physucs. INDIA

Or P Kaw. Inst for Plasma Rosoarch. INDIA

Or. P Rosenas I waed inst of Technotogy. ISPAEL

Loranan In mavona Contor for Theo Physics. ITALY

Miss C Do Paio Assocazione EURATOAHENEA. ITALY

Or G Grosso 1600 to d Fissca del Plasma, ITALY

Prot G Rostanon isuturo Gas lonuzzas Dol Cnr. ITALY

Dr I Yamato Tothido Rer 1 Dover Centor. JAPAN
Prol I Kawakamı. Hroshma Univ. JAPAN

Prol K. Nishikawe. Hroshims UnN, JAPAN

Drector, Japen Alomic Energy Research inst. JAPAN

Prol S Iroh, Kyushu UnN. JAPAN

Rosearch into CU, Navonal Insot to Fusion Scrence, JAPAN

Prot S Tanaka, Kyoto Unur. JAPAN

Lorary, KNo Univ, JAPAN

Prol N Inowe. Univ of Tokyo, JAFAN

Secrotary, Plasma Secton. Eloctorochnical Lab. JAPAN

S. Mon, Tectnica Actusor, MAERI, MAPAN

Dr. O Mitura. Kumemots inst of Tectinotogy. JAPAN

J. Hyeon-Sook, Korea Atomic Enargy Rosearch InsL, KOREA

DI Cha, The Korea Aor Inst of So \& Toch. KOREA

Prol B.S. Liley, UnN of Wakato. NEW ZEALAND

Int of Phrua, Chinose Acad Sa PEOPLE'S REP OF CHINA

Lbrary. Inst a Plasma Phystos. PEOPLES REP OF CHINA

Tinghue UnN Lbray, PEOPLE'S REPUBLIC OF CHINA

$Z$ LI.S W Ins Physa. PEOPLE'S REPUBUC OF CHINA

Prol. J A.C. Cebrod, Instuto Supenor Toanco, PORTUGAL

Or O Rogus, AL I CUZA UnN, ROAMANIA

D. J Villiars, Fusion Studies. AEC, S AFRICA

Prol MA Helloorg. UnN of Natal, S AFRICA

Prol DE Km, Pohang inst of Sa \& Toch. SO KOPEA

Prol CIEMA.T. Fusion Drvision Lbrary, SPAIN

Or L Siontlo. UnN OI UMEA. SWEDEN

Larary, Royal Inst. of Tectmology, SWEDEN

Prot $H$ Withotmeon, Chalmers UnN of Tech. SWEDEN

Contro Phys Des Plasmas, Ecole Polyoch. SWITZERLAND

Bublow. Inst Voor Plasma Fysica. THE NETHEALANDS

Asst Prod Dr S Cakr, Modde East Tech UnN. TURKEY

D. VA. Guktiuh, Sa. Res Inst Electrophys I Apparatus. USSR

O D D Arubr, Siberan Branch of Acadomy of Sa, USSA

O. GA Eisoov, IV Kurchator Inst, USSR

Libranan. The UKS SSR Acadomy of Scconces. USSR

Dr LM Komithmykh, Inst ol Genera Physics, USSR

Komtorctiungsanlege GmbH. Zentralbibliotiek. W GERMANY

Budrothe Inst For Plasmatorschung. W GERMANY

Prol $X$ Schinder, Ruhr. Unnersut Bochum. W GERMANY

D. F Wogner. (ASDEX), Max.Planck-Instutt. W GERMANY

Loranan, Max.Planck-insuur. W GERMANY

Prot RK. Janew. InsL of Ptysics YUGOSLAVIA 

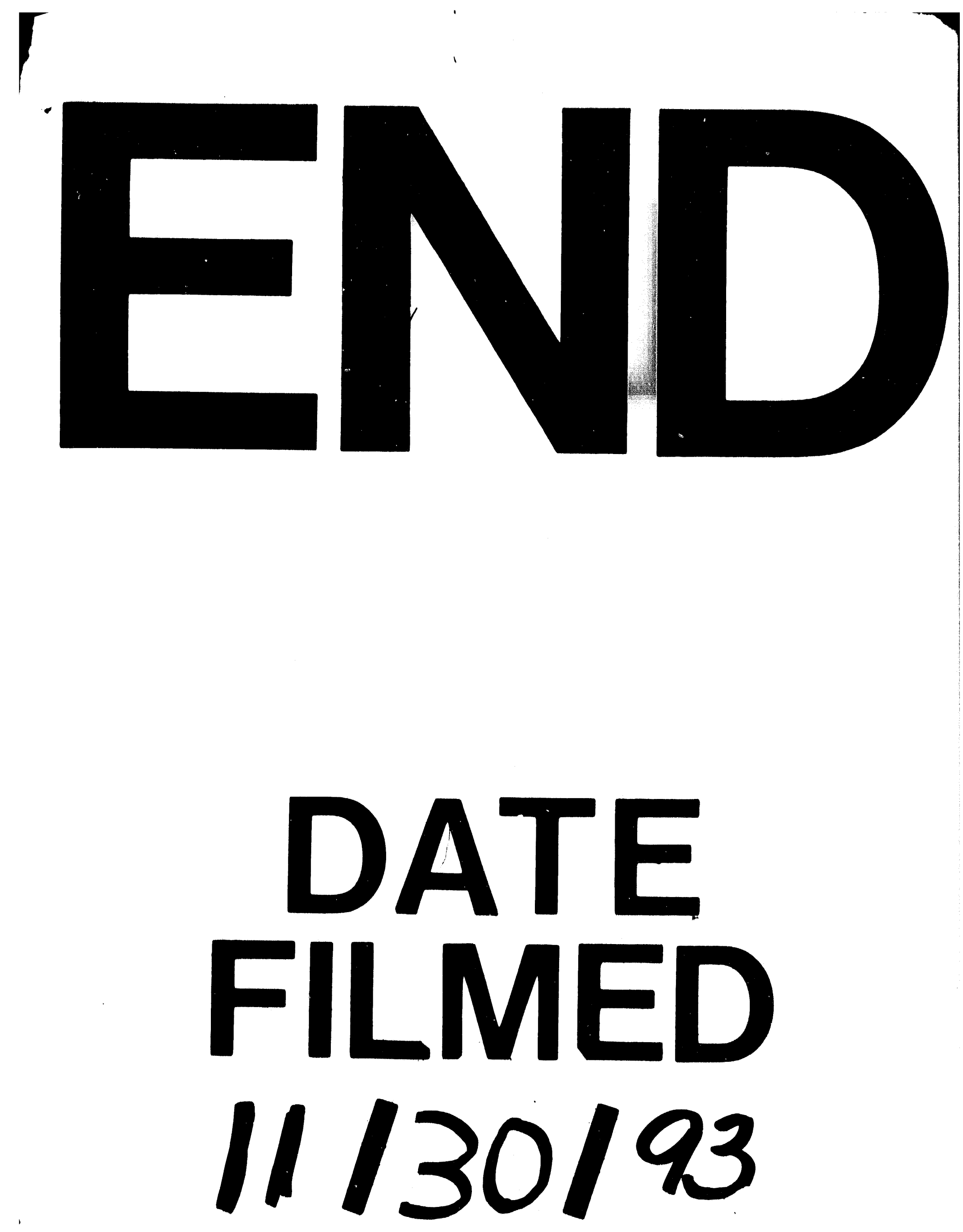
